\title{
The repetitive DNA landscape in Avena (Poaceae): chromosome and genome evolution defined by major repeat classes in whole-genome sequence reads
}

Qing Liu ${ }^{1 *} \mathbb{D}$, Xiaoyu Li ${ }^{1,2}$, Xiangying Zhou ${ }^{1,2}$, Mingzhi Li $i^{3}$, Fengjiao Zhang ${ }^{4}$, Trude Schwarzacher ${ }^{1,5}$ and John Seymour Heslop-Harrison ${ }^{1,5^{*}}$

\begin{abstract}
Background: Repetitive DNA motifs - not coding genetic information and repeated millions to hundreds of times - make up the majority of many genomes. Here, we identify the nature, abundance and organization of all the repetitive DNA families in oats (Avena sativa, $2 n=6 x=42$, AACCDD), a recognized health-food, and its wild relatives.

Results: Whole-genome sequencing followed by k-mer and RepeatExplorer graph-based clustering analyses enabled assessment of repetitive DNA composition in common oat and its wild relatives' genomes. Fluorescence in situ hybridization (FISH)-based karyotypes are developed to understand chromosome and repetitive sequence evolution of common oat. We show that some 200 repeated DNA motifs make up 70\% of the Avena genome, with less than 20 families making up 20\% of the total. Retroelements represent the major component, with Ty3/Gypsy elements representing more than $40 \%$ of all the DNA, nearly three times more abundant than Ty1/Copia elements. DNA transposons are about 5\% of the total, while tandemly repeated, satellite DNA sequences fit into 55 families and represent about $2 \%$ of the genome. The Avena species are monophyletic, but both bioinformatic comparisons of repeats in the different genomes, and in situ hybridization to metaphase chromosomes from the hexaploid species, shows that some repeat families are specific to individual genomes, or the A and D genomes together. Notably, there are terminal regions of many chromosomes showing different repeat families from the rest of the chromosome, suggesting presence of translocations between the genomes.

Conclusions: The relatively small number of repeat families shows there are evolutionary constraints on their nature and amplification, with mechanisms leading to homogenization, while repeat characterization is useful in providing genome markers and to assist with future assemblies of this large genome (c. $4100 \mathrm{Mb}$ in the diploid). The frequency of inter-genomic translocations suggests optimum strategies to exploit genetic variation from diploid oats for improvement of the hexaploid may differ from those used widely in bread wheat.
\end{abstract}

Keywords: Chromosome evolution, Common oat (Avena sativa), Fluorescence in situ hybridization (FISH)-based karyotypes, Genome-specific markers, Intergenomic translocations, Repetitive DNAs

\footnotetext{
* Correspondence: liuqing@scib.ac.cn; phh4@le.ac.uk

${ }^{1}$ Key Laboratory of Plant Resources Conservation and Sustainable Utilization /

Guangdong Provincial Key Laboratory of Applied Botany, South China

Botanical Garden, Chinese Academy of Sciences, Guangzhou, China

Full list of author information is available at the end of the article
}

(c) The Author(s). 2019 Open Access This article is distributed under the terms of the Creative Commons Attribution 4.0 International License (http://creativecommons.org/licenses/by/4.0/), which permits unrestricted use, distribution, and reproduction in any medium, provided you give appropriate credit to the original author(s) and the source, provide a link to the Creative Commons license, and indicate if changes were made. The Creative Commons Public Domain Dedication waiver (http://creativecommons.org/publicdomain/zero/1.0/) applies to the data made available in this article, unless otherwise stated. 


\section{Background}

Genome evolution involves multiple processes including whole genome duplications (WGDs or polyploidy), segmental genome deletions or duplications, chromosome restructuring (fusion, fission, translocation, and inversion), and amplification or loss of gene and repetitive sequences, along with DNA mutation $[1,2]$. There is a growing interest in reconstructing ancestral genomes of fungi [3], animals [4] and plants [5], revealing principles governing genome evolution and diversification leading to speciation and adaptation.

Repetitive DNA constitutes a substantial fraction, typically between 25 and $85 \%$, of plant genomes, and can be referred to as the repeatome. Repeat motifs vary extensively in sequence and dispersion patterns [6-8]. Several major groups of repetitive elements are found: ribosomal DNAs (rDNAs) [both 45S (18S-5.8S-26S) and 5S rDNAs with intergenic spacers], the telomeric repeats, class I retrotransposons (amplified through an RNA intermediate), class II DNA transposons (amplified through DNA copies), and tandem repeats (postulated to be generated/ modified by slippage replication, uneven crossing-over or rolling circle amplification) [7]. Their presence and similarity, variation in copy number and sequences, pose a major challenge to genome assembly and gene annotation [9]. Repetitive DNA has been postulated to have multiple roles in the genome, including genome stability, recombination, chromatin modulation and modification of gene expression [7]. Copy number variations in repeats, representing 5 to $10 \%$ of the human genome, are important for disease and population variation [10].

Through the decades up to 2010, repeatome knowledge came largely from DNA annealing experiments, screens of random clones, restriction fragment analyses, or amplification of conserved elements with primers. Now whole-genome shotgun sequencing approaches can be used for genome-wide, unbiased repeat analysis [11-13]. A k-mer analysis counts the number of motifs k-bases long in whole-genome sequence reads [14], to identify abundant motifs without using reference genomes. The graph-based clustering analysis (e.g. RepeatExplorer $[11,15])$ is another approach to identify and classify repeats from raw reads. Both are de novo identification strategies, and results can be used for repeat identification or protein domain searches. Because of the multiple genomic locations and difficulties of assembly, in situ hybridization to chromosomal preparation is essential to identify the genomic locations and specificity of repetitive motifs [16]. These approaches have been used to quantify the genome repetitive landscape in banana, radish, soybean and tobacco [17-21].

Common oat (Avena sativa L., $2 n=6 x=42$, AACCDD) is a temperate crop (annual production of 23 million tons in 2017; http://faostat.fao.org) with the approved health claim as a 'superfood' because of oat beta-glucan, which helps reduce blood cholesterol level and heart disease risk $[22,23]$. Genomic resource development of common oat, important for breeding and improvement, has lagged behind other major crops [24, 25]. There are several genetic maps of diploid and hexaploid species [26, 27] but no draft genome sequences for the hexaploid crop ( $6 x$ genome size $12,600 \mathrm{Mb} / 1 \mathrm{C}$ ) [28] or its diploid relatives. The oat genome contains numerous families of repeats and apparently frequent chromosome translocations [29, 30]. In recent phylogenetic analyses, common oat was inferred to experience ancient allotetraploidy and recent allohexaploidy events involving $\mathrm{C}$-, A- and D-genome ancestors $[31,32]$, while the genome reshuffling obscures contributions of different candidate maternal A-genome progenitors (bipaternal genome definition referred to [32]).

Here, we aimed to elucidate structure, organization, and relationship of all major repetitive DNA classes in diploid and hexaploid oats, examine their chromosomal locations, and understand the significance of repeatome in genome and chromosome evolution of Avena in the context of genomic, bioinformatic and cytogenetic evidence. The complete picture of repetitive DNAs provides new evidence for events occurring during evolution and speciation in the genus, including hybridization and chromosomal translocation events.

\section{Results}

Graph-based clustering and repeat composition of Avena Raw sequence reads (Illumina $250 \mathrm{bp}$ paired end) obtained from Avena sativa, A. brevis, $A$. hirtula, and $A$. strigosa averaged $43.23 \%$ GC (guanine-cytosine) content (Additional file 1: Figure S1, Additional file 13: Tables S1, Additional file 14: Table S2a). For graph-based clustering of reads, a 1.72 to $2.87 \mathrm{~Gb}$ subset were analysed using RepeatExplorer [11] (Additional file 14: Table S2b). In total, more than $70 \%$ of reads were assigned into just 200 graph clusters of highly related sequence reads (Additional file 2: Figure S2, Table 1), with 12 to 18 clusters (depending on species) representing more than $1 \%$ of all the reads (Additional file 15: Table S3b).

After manual verification by checking domain homology or satellite motifs (Additional file 16: Table S4), $65 \%$ of Avena genome reads were related to transposable elements [TEs, including class I retrotransposons (average $60.80 \%$ ) and class II DNA transposons (average $5.78 \%)$, and tandem repeats [satellite DNA (satDNA), rDNA, and telomere; average 2.70\%] (Table 1, Additional file 17: Table S5 [33]). Long terminal repeat (LTR) retrotransposons dominated in class I retrotransposons, with Ty3-Gypsy (Metaviridae; average 42.8\%; gene order reverse transcriptase-RnaseH-integrase, RTRH-INT) and Ty1-Copia (Pseudoviridae; average 16.6\%; gene order INT-RT-RH) superfamilies, while non-LTR 
Table 1 Repetitive DNA percentage composition by RepeatExplorer analyses of four Avena species genomes

\begin{tabular}{|c|c|c|c|c|c|c|c|c|c|c|}
\hline Species & $\begin{array}{l}\text { Number of clusters } \\
\text { representing }>0.01 \% \\
\text { of genome }\end{array}$ & $\begin{array}{l}\text { Genome } \\
\text { percentage in } \\
>0.01 \% \text { clusters }\end{array}$ & $\begin{array}{l}\text { Genome \% } \\
\text { in top } 15 \\
\text { clusters }\end{array}$ & $\begin{array}{l}\text { Number of } \\
\text { clusters }>1 \% \\
\text { of genome }\end{array}$ & $\begin{array}{l}\text { \% of genome } \\
\text { annotated as } \\
\text { Gypsy }\end{array}$ & Copia & $\begin{array}{l}\text { LINEs non-LTR } \\
\text { retroelements }\end{array}$ & $\begin{array}{l}\text { DNA } \\
\text { trans-posons }\end{array}$ & EnSpm & $\begin{array}{l}\mathrm{GC} \% \text { of } \\
>0.01 \% \\
\text { clusters }\end{array}$ \\
\hline A. sativa & 214 & $71.0 \%$ & $17.8 \%$ & 12 & $43.1 \%$ & $16.0 \%$ & $0.32 \%$ & $5.5 \%$ & $4.0 \%$ & $44.0 \%$ \\
\hline A. brevis & 214 & $71.6 \%$ & $19.5 \%$ & 16 & $42.8 \%$ & $15.9 \%$ & $0.32 \%$ & $5.4 \%$ & $4.0 \%$ & $43.3 \%$ \\
\hline A. hirtula & 198 & $72.5 \%$ & $21.2 \%$ & 14 & $40.5 \%$ & $17.3 \%$ & $0.45 \%$ & $5.9 \%$ & $5.0 \%$ & $44.2 \%$ \\
\hline $\begin{array}{l}\text { A. } \\
\text { strigosa }\end{array}$ & 195 & $71.6 \%$ & $23.7 \%$ & 18 & $40.8 \%$ & $17.4 \%$ & $0.42 \%$ & $5.9 \%$ & $5.2 \%$ & $44.0 \%$ \\
\hline
\end{tabular}

retrotransposons including long interspersed nuclear elements (LINEs) represented less than 0.5\%. Class II DNA transposons, including Helitrons, included up to $5.2 \%$ enhancer suppressor mutator (EnSpm) elements. There were no notable differences of repetitive DNA composition among four Avena genomes (Additional file 17: Table S5). The clustering analysis groups solo-LTRs and SINEs with their parental elements; cluster graphs showed the greater abundance of LTRs compared to the coding sequences (Fig. 1h). The Blast results identified less than 20 chloroplast sequence clusters (abundance ranking 46-168, removed from further analysis), and about 11 rDNA clusters (Additional file 16: Table S4). Only a small proportion (2.44-4.49\%) of clusters were unclassified (Additional file 17: Table S5), showing little similarity to characterized sequences, but some had adenine-thymine-rich domains (Additional file 18: Table S6 [34, 35]). Our analyses were not designed to identify most microsatellite arrays (including telomeric sequences), typically shorter than 10-mers.

Clusters showed characteristic graph patterns (Fig. 2a-p) that we used to classify the repeat families. As examples, $0.15 \%$ of reads formed the star-shape cluster graph for the tandemly repeated satellite DNA As-T119 (Fig. 2h), 0.77\% of reads formed the ray-shape retrotransposon repeat Ah-R31 (Fig. 2n), 0.04\% of reads formed the circular-shape repeat Ab-T159 (Fig. 2o), and 0.02\% of reads forming line-shape simple repeat Ast-R176 (Additional file 19: Table S7). Eight of fragments used for in situ hybridization had 335-360 bp monomers (Additional file 20: Table S8a [36-38]). 312CL151C2 (no PCR products without designation, Additional file 20: Table S8b) was unique as it showed a higher order repeat structure of $232 \mathrm{bp}$ dimer consisting of two closely related $116 \mathrm{bp}$ monomers.

\section{k-mer analyses of Avena}

For cumulative repetitivity frequency plots of 10 - to 64-mers, the steeper slope indicated the faster cumulative percentage changes, which varied relatively gentle for short k-mers (10- to 17-mers) and gradually increased steep-slope for longer k-mers (18- to 64-mers) (Fig. 2a-c). For the same repetitivity frequency, a shorter k-mer motif has a higher cumulative percentage (and higher frequency in raw reads; Fig. 3a-c). Among the four Avena species, 16-mer motifs occurring $\geq 10$ times accounted for $44 \%$ of $A$. sativa genome, somewhat higher than other species (28\% of $A$. brevis, $34 \%$ of $A$. hirtula, and $40 \%$ of $A$. strigosa; Fig. $3 \mathrm{~d}$ ). The 64-mer motifs occurring $\geq 10$ times accounted for $11 \%$ of $A$. strigosa genome (Fig. 3e), higher than other species (5\% of $A$. sativa, $2 \%$ of $A$. brevis, and $4 \%$ of $A$. hirtula) genomes. Overall, the graphs were consistent with the RepeatExplorer analysis, with a group of very abundant sequences representing about a quarter of the genome (inflection in e.g. the 16-mer graph, Fig. 3d), and other abundant sequences representing about $70 \%$, before a flatter region of the graph with motifs represented less than 10 times per genome. For 16-mer motifs occurring $\geq 10$ times, the cumulative percentage of common oat was nearly equivalent to Petunia axillaris, followed by $A$. strigosa, sorghum, $A$. hirtula, A. brevis, tomato, and potato (Fig. 3f [21, 39-41]); it was notable that the cumulative percentage of 16-mer motifs occurring $\geq 1000$ times converged for Avena species, tomato, and potato.

\section{Chromosomal location and genome specificity of highly repetitive motifs Repetitive fragments used as FISH probes}

To localise repeats on Avena sativa chromosomes (Figs. 4 and 5, Additional files 3-10: Figures S3-S10), 25 probes were designed from representative sequences identified by k-mer and RepeatExplorer analyses to use for in situ hybridization, including nine satellite, one DNA transposon, four LTR-Gypsy retroelements and eleven unclassified sequences (Additional file 19: Table S7). Except for AF226603_45bp and pAs120a [36, 37], we selected sequences with little or no homology to repetitive elements (TEs or tandem repeats) previously isolated by PCR or cloning strategies [30,42]. 45S and 5S rDNA $[34,35]$ were used to identify some chromosomes (Additional file 18: Table S6).

Copy numbers and relative proportion of the selected probes were analysed in silico in A. sativa and three A-genome diploids (Additional file 20: Table S8a) to check abundance and genome specificity. Most repeats (92\%) were present in all four genomes (Additional file 20: Table S8a), with expected variation from over 3 million copies per genome (As-16mer43bp in $A$. 


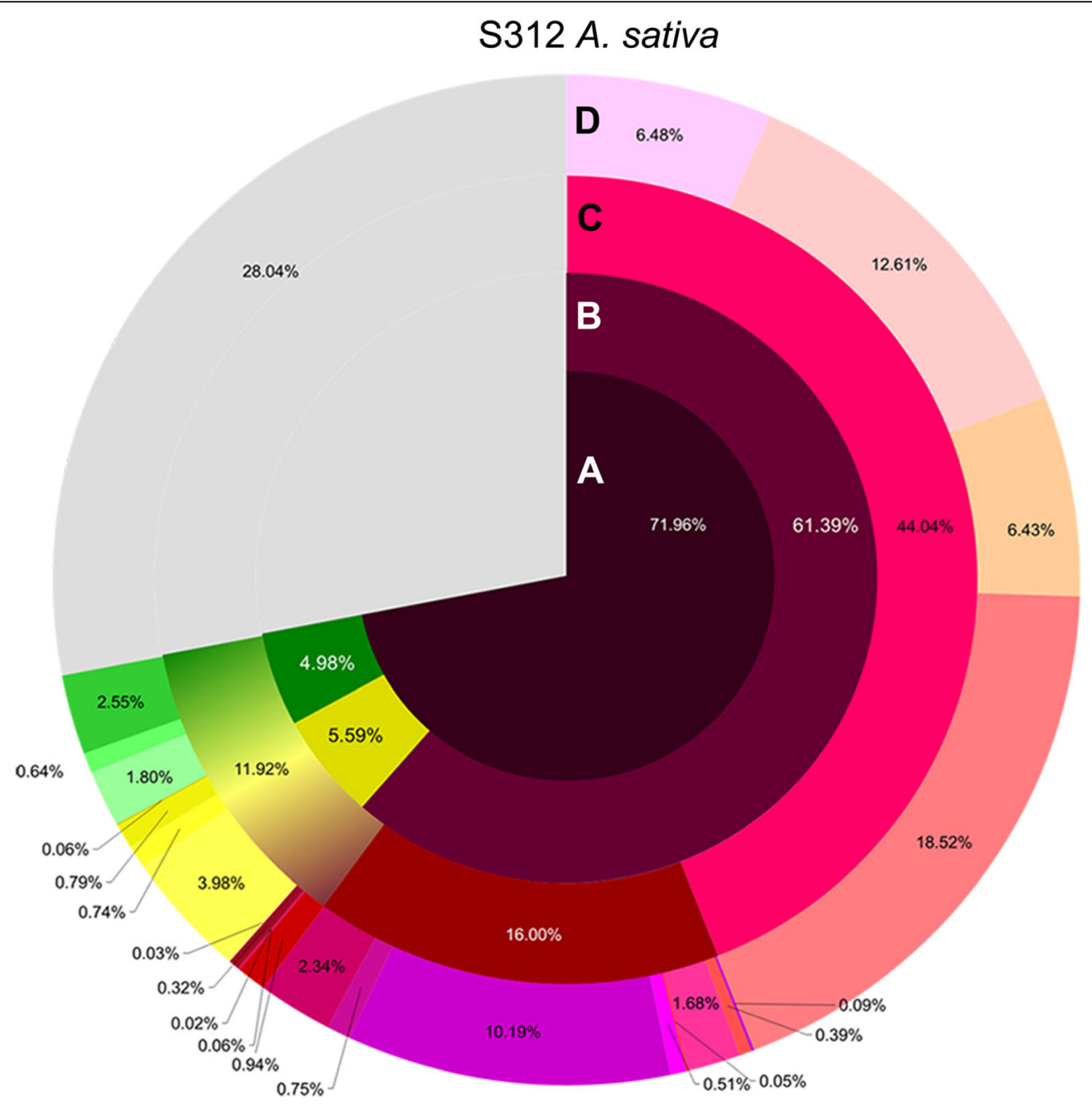

From inside
A Total repeats
Gene and regulatory sequences
B - Retrotransposons
Transposons
Non-retrotransposon and non-transposon repeats
C Total Gypsy
- Total Copia
D Gypsy Ogre/Tat
Chromovirus
11 Non-Gypsy and non-Copia repeats
- Unclassified Gypsy
- Tork
Athila
- Maximus/SIRE
- Ivana/Oryco
TAR
- Alel-Retrofit
Bianca
Angela
PARA-RT
- Cassandra
LINE L1
MULE-MUDR PIF_Harbinger
Unclassified Copia
- Unclassified LTR
TcMar-Stowaway
CMC-EnSpm
Satellite/telomere
rDNA
Helitron
Unclassified repeats
(Low complex, simple repeats, unknown)

Fig. 1 Frequency of major repetitive DNA classes in Avena sativa. Repeats were identified by graph-based clustering (RepeatExplorer) and in abundant k-mer motifs, and classified by nucleotide domain hits and database Blast searches. Concentric circles show increasing information from center; results were similar for diploid genomes (Additional file 17: Table S5) 

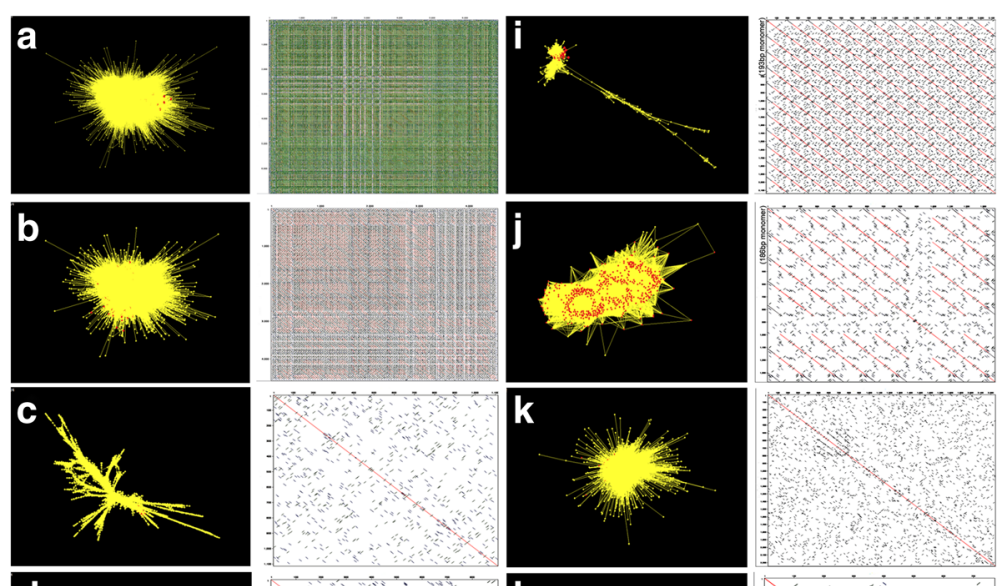

d
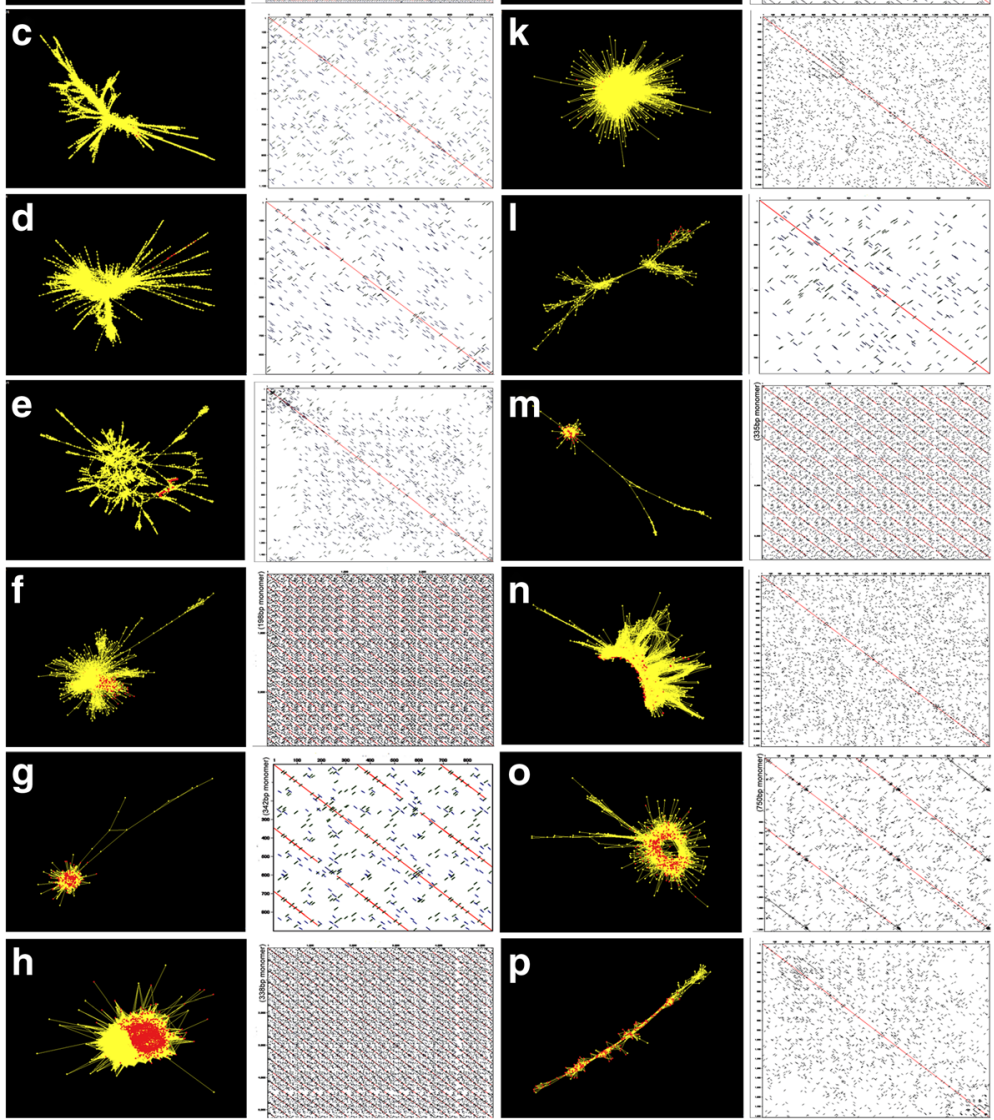

Fig. 2 Repeat cluster graphs and dotplots of selected repeats in Avena species. For each repeat, the RepeatExplorer cluster is shown (left; yellow nodes represent all assembled contigs within the repeat cluster; red nodes represent members of the contig analysed in greater detail, including by amplification and in situ hybridization. A self-dotplot of the selected contig is shown in the right panel; parallel diagonals show tandem repeats. a As_16mer43bp, identified in cluster 289CL8C154. b AF226603_45bp in cluster 289CL8C248. c Cluster Ab-R18. d Ab-R19. e Ast-R87. f Ab-T145. g Ah-T118. h As-T119. i As-T153. j As-T175. k pAs120a in cluster 312CL17C141. I Ast-R171. m Ast-T116. $\mathbf{n}$ Ah-R31. o Ab-T159. p Ast-R176. Repeat names include species origin of the exemplar family member: Ab, Avena brevis; Ah, A. hirtula; Ast, A. strigosa; As, A. sativa and repeat type: T, tandem; R, retrotransposon

sativa) to being almost undetectable. Eight of the fragments used for in situ hybridization had 335-360 bp monomers (Additional file 18: Tables S6, Additional file 19: Table S7, Additional file 20: Table S8a). The monomer number shown in dotplots (Fig. 2f, j, $m$ and o) was a consequence of variability between monomers and clustering algorithm, and not related to genome structures: tandem repeat counts require very long reads (e.g. Nanopore or PacBio Sequel) or chromosome walking (e.g. BAC clones). Repeat copy numbers in the three diploid A genome species analysed was not the same (Fig. 6a), showing the whole spectrum of distribution and indicating differential amplification or loss after evolutionary separation.

No repeat was predominant in A. strigosa, a species where repeats As-T153 and As-16mer43bp were also absent. One repeat family (Ast-R171) was much more abundant in A. hirtula, and four families (As-T153, Ab-T166, Ast-T125 and Ab-T145) were dominant in the A. brevis genome (Fig. 6a, Additional file 20: Table S8a). The As-16mer43bp repeat was only abundant in A. brevis, being absent in A. strigosa and only 260 copies in A. hirtula. 


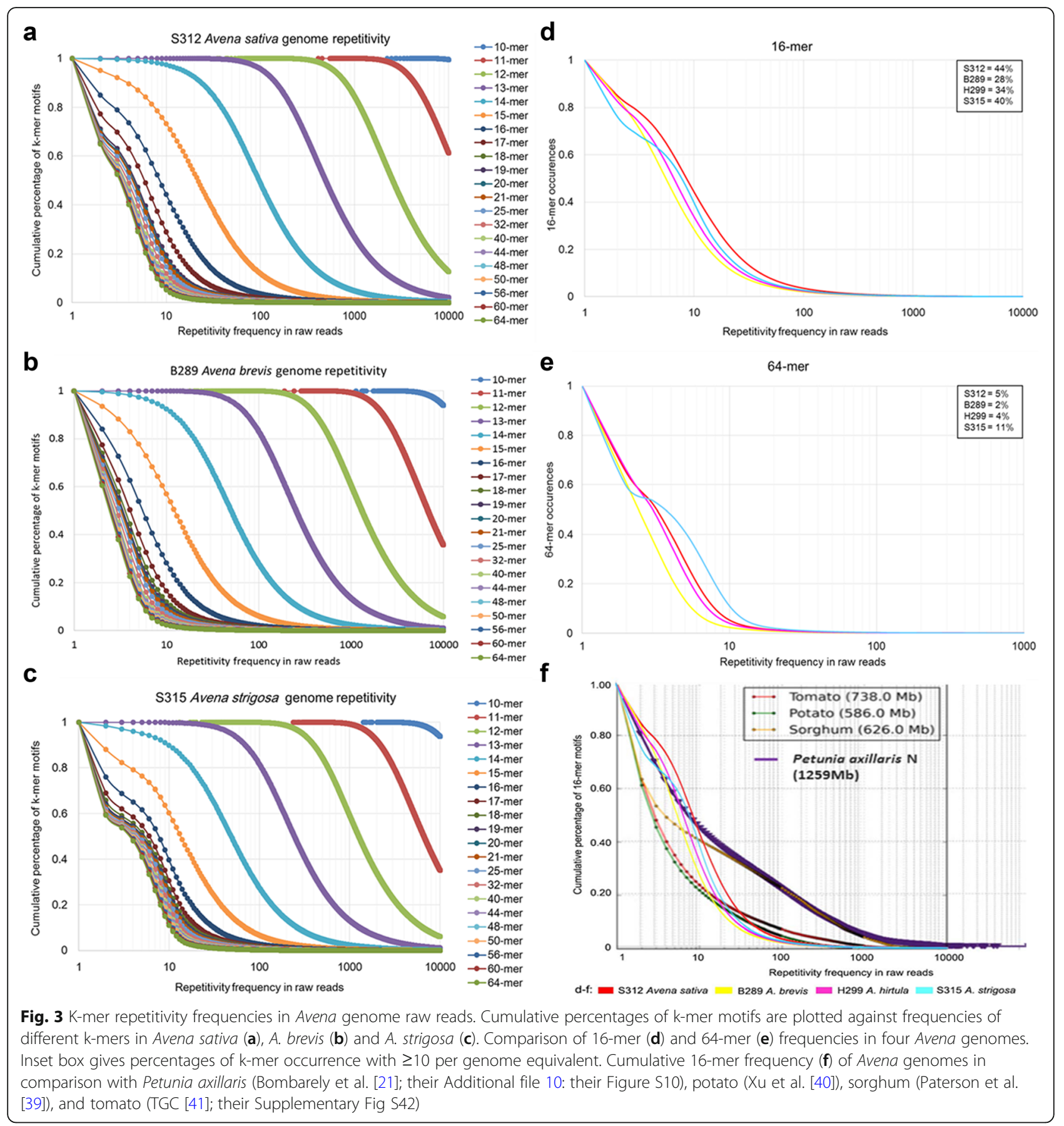

For in situ hybridization, sequence fragments were synthesized as end-labelled oligonucleotides, or amplified by PCR from genomic DNA of Avena (A-genome species $A$. brevis, A. hirtula, A. strigosa, A. atlantica, A. wiestii, and $A$. longiglumis and $\mathrm{C}$-genome species $A$. eriantha; Additional file 18: Table S6). Results from in situ hybridization of 25 repetitive sequences identified here to A. sativa metaphase chromosomes using two or three probes simultaneously are summarized in Additional file 21: Table S9,
Figs. 4 and 5, Additional files 3-10: Figures S3-S10). As predicted from k-mer and RepeatExplorer analyses (Additional file 20: Table S8a), all probes gave hybridization signals and signal strength was generally in accordance with in silico copy number estimated.

Avena sativa chromosomes were assigned to genomes based on karyotype [43] and probe knowledge (particularly the A-genome specific probe pAs120a [35], C-genome specific AF226603_45bp [37], 45S and 5S 

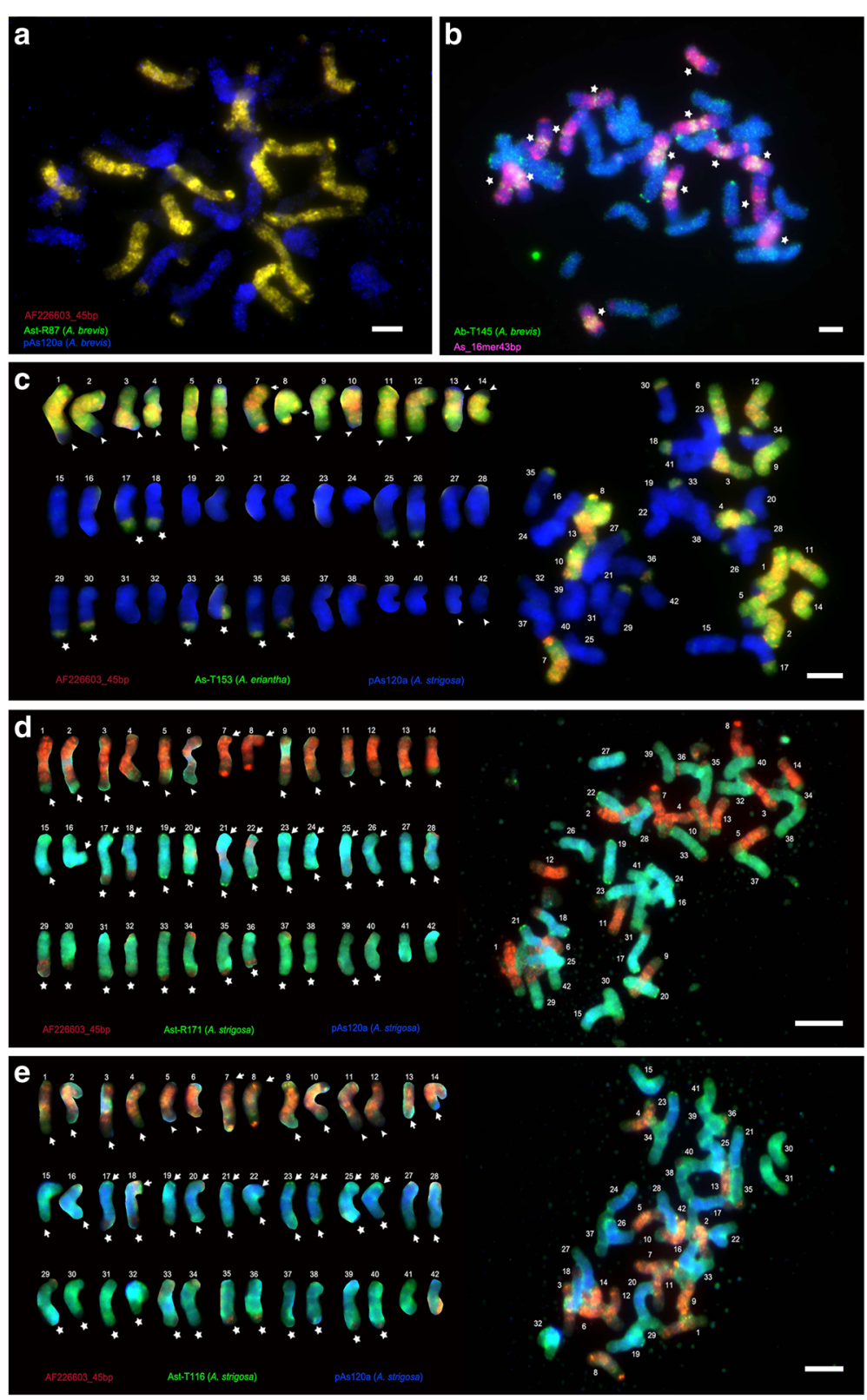

Fig. 4 Localization of repetitive sequences on A. sativa metaphase chromosomes by in situ hybridization. Root tip chromosomes fluoresce cyan with DAPI (UV excitation) stain; probe hybridization sites are detected by fluorescence shown in red, green and/or blue (false colour from near-infra-red fluorescence). (A-C) C-genome specific probes; (D-E) D-genome specific probes. a Probes AF226603_45bp (red), Ast-R87 (green; yellow where probes overlapping) and pAs120a (blue) from A. brevis. b Probes As_16mer43bp (red) and Ab-T145 (green). (c-e) Metaphases (right) and chromosomes arranged as karyotypes showing genome origin (left) with chromosomes approximately paired by probe pattern and size (Chromosomes 1-14 Cgenome; 15-28 A-genome; 29-42 D-genome). White asterisks, arrows, and arrowheads indicate notable C-, A-, and D-chromosome signals. c Probes AF226603_45bp (red), As-T153 (amplified from A. eriantha; green) and pAs120a (blue). d Probes AF226603_45bp (red), Ast-R171 (green), and pAs120a (blue) both amplified from A. strigosa. e Probes AF226603_45bp (red), Ast-T116 (green), and pAs120a (blue). Scale bars =5 $\mu \mathrm{m}$

rDNA). Chromosomes were numbered by descending order of sizes and arranged in pairs using morphology and hybridization patterns: chromosomes 1-14, 15-28 and 29-42 belong to C-, A-, D-genomes, respectively (Figs. 4c-e and 5a-c). Both in situ hybridization patterns and bioinformatic copy number counting allowed us to classify repeat sequences into five categories depending on genome specificity: C-, A-, and D-genome specific repeats showed stronger hybridization to chromosomes of one genome (Fig. 4a-e, Additional files 4, 5 and 6: Figures S4-S6); additional repeats were $\mathrm{A}+\mathrm{D}$-genome specific or present with similar strength in all three genomes (Fig. 5a-e, Additional file 7, 8 and 9: Figures S7-S9). 

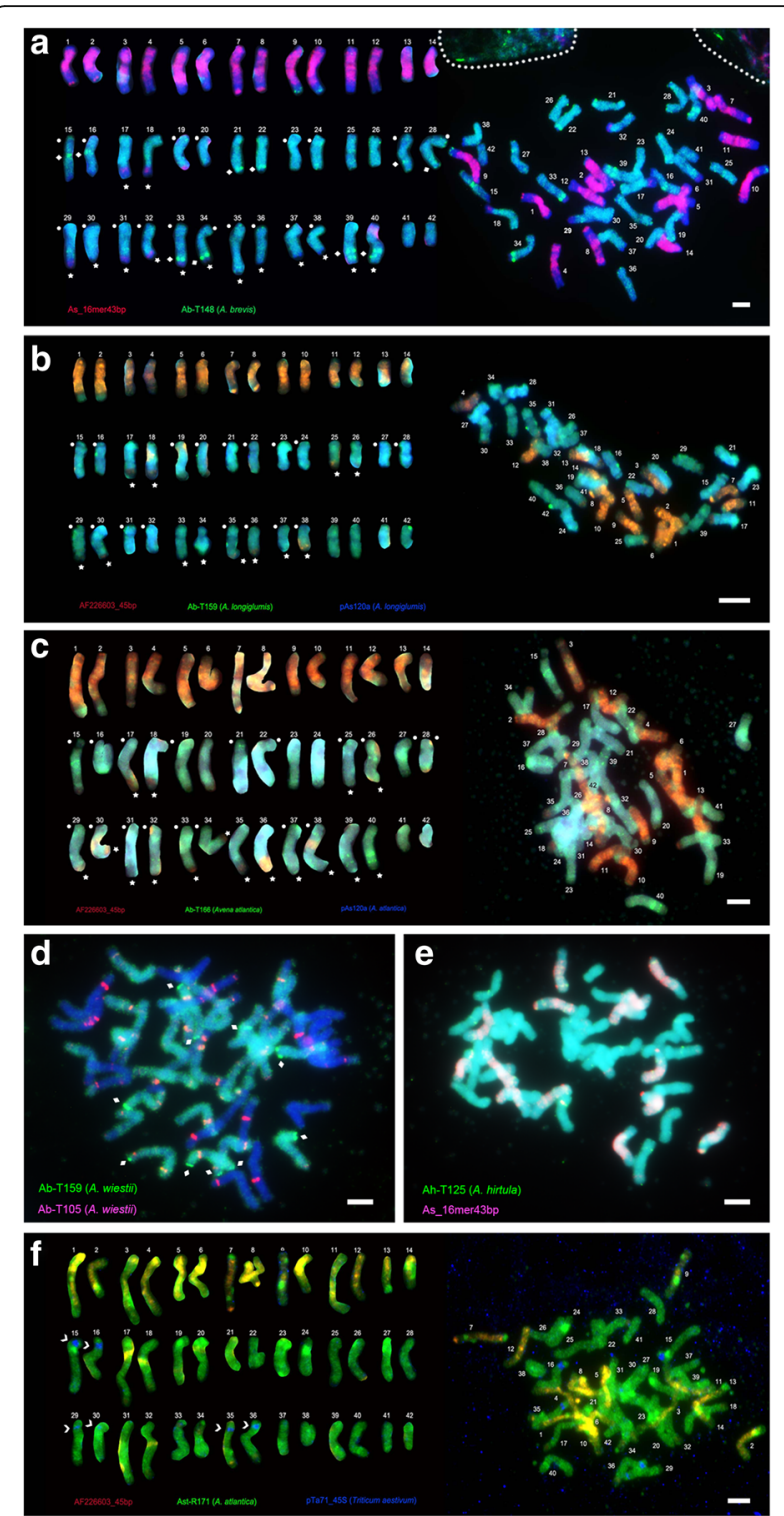

Fig. 5 Localization of repetitive sequences on A. sativa metaphase chromosomes. Details of colours and arrangements as Fig. 4. a Probe As_16mer43bp (red) and A + D-genome specific probe AbT148 (green). b Probes AF226603_45bp (red), pAs120a (blue) and A + D-genome specific probe Ab-T159 (green). c AF226603_45bp (red) A/D-genome specific Ab-T166 (green), and pAs120a from A. atlantica. (blue). (F) Probes AF226603_45bp (red), Ab-T148 (green) from A. atlantica and $45 \mathrm{~S}$ rDNA probe pTa71 (blue, denoted by white angle brackets). d Probes Ab-T159 (green) and Ab-T105 from A. wiestii. (red). e Probes Ah-T125 (green) from A. hirtula and As_16mer43bp (red). Scale bars $=5 \mu \mathrm{m}$

\section{C-genome specific repeats}

Ten non-homologous in situ hybridization signals were detected at intercalary, pericentromeric, and subtelomeric regions on $14 \mathrm{C}$-genome-origin chromosomes. Four unlabelled terminal regions we seen on pairs 5/6 \& 11/12 indicative of C-D and C-A translocations, respectively (Fig. 4d-e, Additional file Additional files 4, 5 and 6: Figures S4-S6).

Repeat As_16mer43bp is highly abundant with over 3 million copies, representing $1.45 \%$ of the Avena sativa genome (Fig. 2a, Additional file 20: Table S8a; a synthetic labelled oligonucleotide was used as probe), and showed strong signals, being dispersed along all $\mathrm{C}$-genome chromosome arms with stronger signals at most pericentromeric regions, and weak dispersed signals on distal regions of $14 \mathrm{~A}$ or D-chromosome long arms (17/18 \& 29-40; Fig. 5a). The less abundant repeat AF226603_45bp $(0.33 \%$ of A. sativa genome; Additional file 20: Table S8a) showed a similar distribution pattern: abundant on $14 \mathrm{C}$-chromosomes $(755,507$ copies; Fig. 2b, Additional file 20: Table S8a), with dispersed signals along arms and pericentromeric regions except for ends of 12 C-chromosomes 1-6 \& 9-14 (Fig. 4c-e). AF226603_45bp signals were also present at the long arm terminals of $10 \mathrm{~A}+\mathrm{D}$-chromosomes with distinctive signals on nearly half of pair 17/18 long arm and one third in chromosomes 25/26, 29-40 (Fig. 4c-e, Additional file 4: Figure S4a-4f, Additional file 5: Figure S5a-S5f Additional file 6: Figure S6a-S6 f). Additionally, nucleolus organizer regions (NORs) were observed using probes Ab-T148, Ab-T159, and Ast-T116 on 20 A + D-chromosomes 15/16, 19-24 \& 27-38 (Figs. 4e and $5 \mathrm{a}, \mathrm{b}$, Additional file 5: Figure S5a-S5f, Additional file 9: Figure S9a-S9f).

Several retrotransposon repeats, Ab-R18, Ab-R19 and Ast-R87 (Fig. 2c, d and e), but also tandem repeats Ab-T145, As-T153 and Ah-T118 (Fig. 2f, g and i) showed dispersed signals, with high abundance on 14 C-chromosomes (Fig. 4b, Additional file 3: Figure S3a-S3b) and much less or no signals on A- and D-chromosomes (Fig. 4a and c).

Other probes labelled only some C-genome chromosomes and showed additionally more uniform signals on all chromosomes indicating large tandem arrays of at least $20 \mathrm{~kb}$ to see FISH signals as double- or more-dots (Additional file 3: Figure S3d and S3e), e.g. As-T175 and As-T119 (Fig. 2h and j).

\section{A-genome specific repeats}

Retrotransposon-related probes (RepeatExplorer Clusters 289CL22, 299CL11, 312CL17 and 315CL22 harboured homologous sequences to the published pAs120a [36]; Fig. 2k). In situ hybridization with probes from all six diploids showed similar and uniform signals on all A-chromosomes with several ends missing, but additional signals at ends of some other chromosomes indicating A-C (17/18 \& 25/26; Fig. 4c) and A-D (15/16, 19-24 \& 27/28; Fig. 4d, e, Additional file 4: Figure S4a-S4f, 


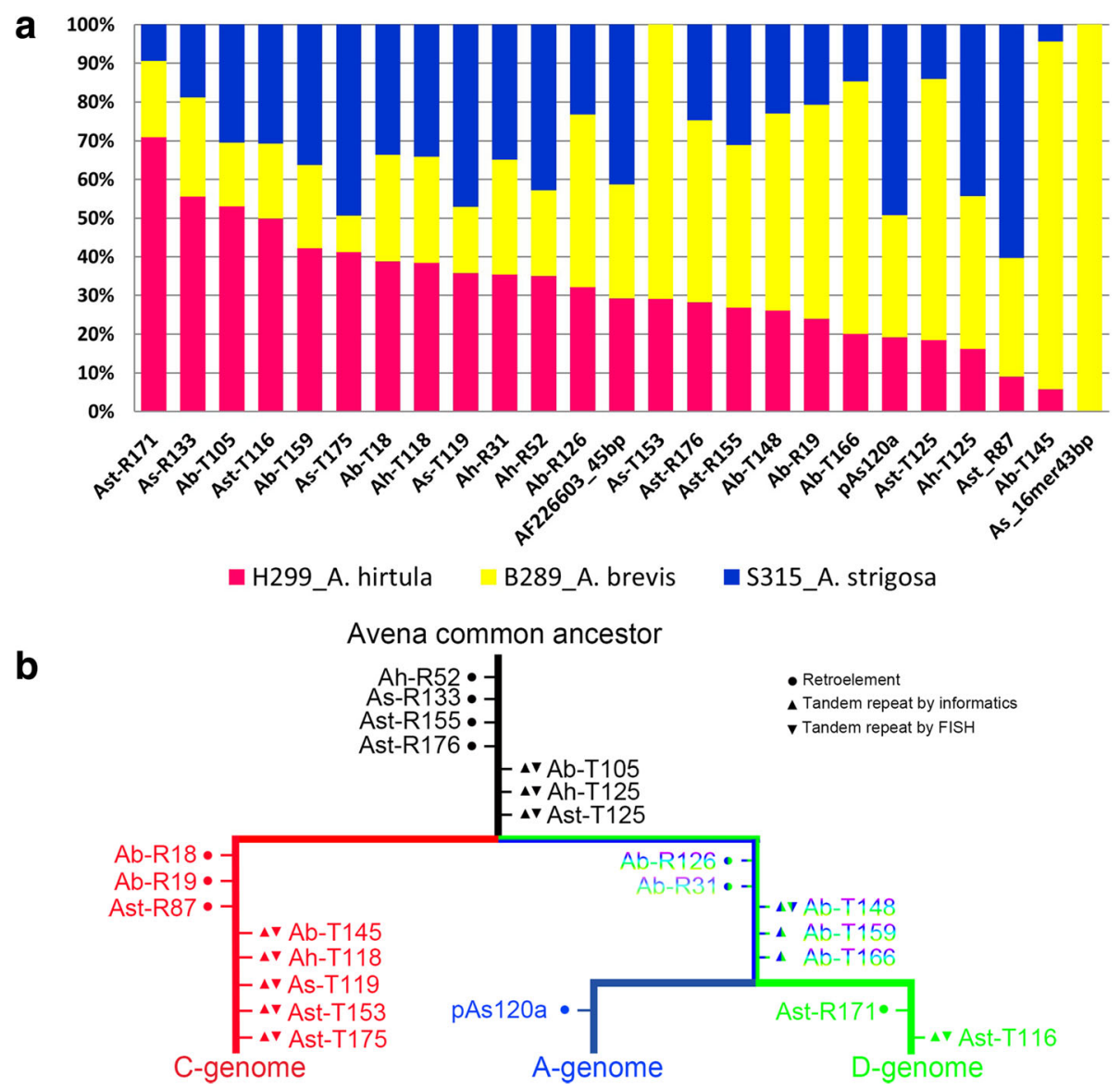

Fig. 6 Relative proportions and an evolutionary model of repetitive DNA motifs in common oat genomes. a Relative proportion of repeats in A. hirtula, A. brevis, and A. strigosa genomes denoted by pink, yellow, and blue columns, respectively. $\mathbf{b}$ Evolutionary model of repetitive DNA motifs. Red, blue, and light green characters denote $C_{-}, \mathrm{A}-$, and D-genome specific repeats, respectively; blue-green gradient characters denote A + Dgenome specific repeats, and black characters denote FISH probes labelling C-, A- and D-genomes. Ab, A. brevis; Ah, A. hirtula; Ast, A. strigosa; As, A. sativa. T, tandem; R, retrotransposon. $\bullet$ : retroelement; $\mathbf{\Lambda}$ : tandem repeat verified by bioinformatics; $\mathbf{\nabla}$ : tandem repeats verified by in situ hybridization; NCBI blast results of tandem repeat families see Additional files 22: Table S10

Additional file 5: Figure S5b-S5c and S5e-S5f) translocations.

\section{D-genome specific repeats}

Retrotransposon Ast-R171 (Fig. 2l) and tandem repeat Ast-T116 (Fig. 2m) showed strong signals predominantly on D-chromosomes (Fig. 4d, e, Additional files 4-5: Figures. S4a-S4f and S5a-S5f), with several bands on A-chromosomes and minimal signals on Cchromosomes (Additional file 4: Figure S4b-S4c and S4e-S4f, Additional file 5: Figure S5b-S5c and S5e-S5f). Ast-R171 showed D-C translocations with subterminal double-dot signals on $12 \mathrm{D}$-chromosomes (29-40; Additional file 4: Figure S4a-S4f), but missing terminal signals on $12 \mathrm{D}$-chromosomes (29-40) that in turn show $\mathrm{C}$ genomic repeats (Fig. 4d, Additional file 4: Figure S4a-S4f). Similarly, Ast-T116 was missing from 12 D-chromosome long arm terminals (chromosomes 2940; Additional file 5: Figure S5a-S5f) that either showed
A- or C-genome-specificity. Ast-T116 showed typical signals of tandem repeats, i.e., double-dots on one or both terminals of A- chromosomes 17-26 (Fig. 4e, Additional file 5: Figure S5b and S5e-S5f). Sometimes, weak translocation signals were observed on long arm terminals of chromosomes 31/32 \& 37-40 in Fig. 4d and Additional file 6: Figure S6a-S6c, and 29-34 \& 37/38 in Fig. 4e and Additional file 6: Figure S6d-S6 f.

\section{$A+D$-genome specific repeats and repeats labelling $C_{-}, A-$ and $D$ - genomes}

Signals of five in situ hybridization probes were almost uniform on $\mathrm{A}+\mathrm{D}$-chromosomes but relatively weak on C-chromosomes (Fig. 5a-d, Additional files 7, 8 and 9: Figures S7-S9). Probes for a retrotransposon Ab-R126, Ah-R31 (Fig. 2n), and tandem repeat Ab-T148 produced dispersed signals on $\mathrm{A}+\mathrm{D}$-chromosomes with 16 unlabelled A + D-chromosome terminals (17/18, 25/26 \& 29-40; Additional files 7, 8 and 9: Figures S7-S9), and 
some unevenly intercalary bands across A $+\mathrm{D}$-chromosome arms (Fig. 5a, Additional file 7: Figure S7e, Additional file 8: Figure S8e, Additional file 9: Figure S9c-S9e). Tandem repeat Ab-T159 (Fig. 2o) and Ab-T166 showed evenly dispersed and weak pericentromeric band signals on $\mathrm{A}+\mathrm{D}$-chromosomes; intergenomic translocations were observed on $12 \mathrm{~A}+$ D-chromosome terminals $(17 / 18,25 / 26,29-32$ \& 3538 ) and on 12 C-chromosome terminals (1-6 \& 914), with dispersed signals on $\mathrm{A}+\mathrm{D}$-chromosomes like retrotransposons (Fig. 5b-d, Additional file 6: Figure S6i).

Seven probes showed dispersed signals along C-, Aand D-chromosomes (Additional file 21: Table S9). Retrotransposons Ah-R52 and repeats As-R133, Ah-T125, Ast-R155 and Ast-R176 showed dispersed signals on C-, A- and D-chromosomes (Figs. 2p and 5d-e, Additional file 10: Figure S10a-S10f). Sequence Ab-T105 and Ast-T125 showed intercalary double-dots on C-, A- and D-chromosomes (Fig. 5d, Additional file 10: Figure S10f).

\section{rDNA sites}

$45 \mathrm{~S}$ and $5 \mathrm{~S}$ rDNA sequences occupied about $0.5 \%$ of the Avena genome (Additional file 17: Table S5). Tandem repeat $45 \mathrm{~S}$ rDNA (9059 bp) contained $3197 \mathrm{bp}, 1811 \mathrm{bp}$, $260 \mathrm{bp}, 168 \mathrm{bp}, 216 \mathrm{bp}$ and $3407 \mathrm{bp}$ of ETS, 18S, ITS1, $5.8 \mathrm{~S}$, ITS2 and $26 \mathrm{~S}$, respectively, while tandem repeat $5 \mathrm{~S}$ rDNAs (544 bp) contained $180 \mathrm{bp}$ coding sequences and 158-206 bp intergenic spacers (Additional file 11: Figure S11a-S11b). Six 45S rDNA loci were identified on six NORs of A and D-chromosomes (15/16, 29/30 \& 35/36), and fourteen $5 \mathrm{~S}$ rDNA loci were identified on six NORs plus eight intercalary positions of A and D-chromosomes (15/16, 29/30, 31/32 \& 35/36), respectively (Fig. 5f, Additional file 11: Figure S11c-S11e). The 5S rDNA signals collocalized with $45 \mathrm{~S}$ signals on six NORs of A- and D-chromosomes (Fig. 5f, Additional file 11: Figure S11e), plus extra pairs of $5 \mathrm{~S}$ intercalary signals located on pair 31/32 (Additional file 11: Figure S11d-S11e). Extended chromatin and hybridization signals were visible on six NOR A- and D-chromosomes (Additional file 11: Figure S11e) indicating the NOR-rDNA transcription was more active on A- and D-compared to C-chromosomes.

\section{Discussion}

\section{Identification and abundance of repetitive DNAs Genome wide}

Analysis of unprocessed Avena genomic DNA sequence reads using motif counting (k-mer analysis) and graphbased clustering shows that repetitive DNA sequences represent some $72 \%$ of the genome (Fig. 1, Table 1).
Combining the in silico analysis with molecular cytogenetics on chromosomes in situ, we could identify the nature of the motifs and measure their abundance to give a comprehensive survey and evolutionary relationships of the repeat landscape of oat (Figs. 1 and 6). Notably, 96\% of the sequences examined here could be classified as being related to either transposable elements or a relatively small number of tandemly repeated motifs (Figs. 1 and 2). Our strategy would not expect to reveal microsatellite motifs, short runs of dinucleotide or trinucleotide repeats with unique flanking regions, known to have an uneven distribution across the genome [44]. While there are increasing reports of genome-wide repeat surveys $[13,45$, 46], most sequence assemblies collapse repeats to variable extents [21, 47], while library screening or PCR amplification with primers are selective. Thus detailed comparisons between our results and many published analyses using whole genome assemblies, reference repeats (e.g. RepeatMasker), or targeted screening may not be valid. Furthermore, classification of "families" within major groups of repeats is flexible, with some distinct families, and others where there are intermediates between sequences that would otherwise be regarded as distinct. Many of the major families of repeats identified here have been identified previously in selective screens of DNA libraries [30, 36], although these studies could not quantify their abundance in the various diploid and the hexaploid genomes. Importantly, unlike the analysis of unprocessed random reads here, selective screens cannot show that all the repetitive components of the genome have been surveyed.

The 16-mers identified by our k-mer analysis with more than 10 copies per genome correspond to the figures from potato and tomato (see Fig. 3f). The 16-mers occurring less than 10 times represented between 24 and $40 \%$ of the oat genome (Fig. 3), indicate a relatively high variation within repeat sequence motifs, and these families may not be detected by reassociation kinetics (experimental) or graph-clustering (bioinformatics). Overall, the proportion of 16-mers occurring 10 or less times in Avena is similar to the $30 \%$ in Petunia or Sorghum (Fig. 3f). However, the variation between four Avena species (24, 28, 34 and 40\%; Fig. 3) is hard to explain but may suggest greater homogenization in A. strigosa. A change in slope, as seen in A. strigosa for k-mers longer than $16 \mathrm{bp}$ (Fig. 3), could be related to the frequencies of different repetitive DNA classes or their homogenization, but there were no conspicuous differences in repeat classes between $A$. strigosa and the other diploids (Additional file 14: Table S2). A. sativa shows a weaker change in graph slope (Fig. 3), consistent with addition of the genomes from one A. strigo$s a$-like and two other species. LTR retrotransposons are largely responsible for the dramatic differences in genome sizes between related plant species, e.g. six-fold size 
difference between maize and rice genomes [48, 49] so they could equally play a role here.

\section{Transposable elements}

Major families of LTR retrotransposons represented 60\% of the genome and $85 \%$ of the repeatome in Avena (Fig. 2), a value that varies widely in different species. In Brachypodium distachyon (genome size $272 \mathrm{Mb}$ ) retrotransposons represent $21.6 \%$ of the genome or $26 \%$ in rice $(430 \mathrm{Mb}$ [50]), or $85 \%$ in bread wheat $(6 x, 16,000 \mathrm{Mb}$ [51]) and 'over $80 \%$ ' reported in barley $(2 x=14,5100 \mathrm{Mb}$ [46]). In Avena, Ty3-Gypsy like LTR-retrotransposons (retroelements) were 2.3 to 2.7 times more abundant than Ty1-Copia like sequences (Figs. 1 and 2, Additional file 17: Table S5). These values are closely similar to the three wheat genomes (ratio 2.5 to 2.9 [51]), although differs in other species: total repetitive DNA occupies $73 \%$ of the Coix aquatica genome (1.7 Mb), but the Gypsy/Copia is only 0.74 [52]. DNA transposons, shorter in element length than retroelements, represented 5.5\% of the Avena genome, with CMC-EnSpm like sequences most abundant. This relatively high value is similar to Brassica oleracea HDEM (4.69\% [53]) and Petunia species (4.64-5.21\% [21]), although other species are much lower including another Brassica, B. rapa Z1 (1.72\% [53]), and the Solanaceous Solanum and Nicotiana species $(0.6-1.51 \%[21])$.

All genomes have mechanisms controlling TE amplification. Schorn et al. [54] have shown in Arabidopsis how RNA-driven DNA methylation is responsible for silencing, as is most likely the case for pararetrovirus sequences [55]. Large genomes bear higher proportions of TE sequence, and Lyu et al. [56] suggested that TE load reduction is the most important driver of genome diminution in mangroves. Here, it is notable that oat retrotransposon-related repetitive sequences families vary in abundance between diploid species, and some are essentially specific to one or two of the genomes (Fig. 6), suggesting loss and gain of particular families and directed turnover.

\section{Tandem/satellite repeats}

Tandem repeats or satellite DNA is a feature of most eukaryotic genomes. Here, we found 12 tandem repeat families, in eight of which the monomer lengths were $335 \mathrm{bp}-360 \mathrm{bp}$ (Additional file 22: Table S10). This has been noted as a monomer length required to wrap around two nucleosomes ( $150 \mathrm{bp}$ DNA for a single nucleosome) spaced by a variable unwrapping linker region of 30-60 bp [16, 57]. Structural interactions between nucleosomes and DNA repeats can impact chromatin dynamics $[58,59]$ and the stable wrapping of tandem repeats could be important for genome stability and methylation of domains leading to silencing. Tandem repeat probes show discrete signals on common oat chromosome arms (Additional file 3: Figure S3d-3e, Additional file 5: Figure S5e-S5f) indicating large arrays of at least $20 \mathrm{~kb}$, but some also show dispersed signals at intercalary sites (Fig. 5b-d), likely to represent multiple smaller arrays.

Submotifs of a repeat family can be used as genome-specific probes for in situ hybridization, e.g. the Brassica C-genome specific CACTA transposon [60]. Here, probe As_16mer43bp motif was found 3,346,757 reads in $A$. sativa, but was absent in $A$. strigosa (Additional file 20: Table S8a). Another repeat AF226603_45bp motif was first identified by Southern blot analysis [61] as being abundant. In contrast, two short $45 \mathrm{bp}$ motifs of unknown family produced uniformly dispersed signals on common oat C-chromosomes (Fig. 4a, c-e). They are a relatively unusual length for plant repeat motifs, although c. 60 bp-length minisatellites are common in mammals [62].

rDNA was used as chromosome-specific probes. Based on phylogenetic evidence, in Avena, two NORs (45S rDNA sites) per haploid chromosome set were ancestral characters, while chromosome complements with 4 or more NORs were derived characters [63]. Structurally, the elimination of C-genome rDNAs and partial elimination of A-genome rDNAs following a hexaploidization event in A. sativa indicates that rDNA from one ancestor (the paternal genome (see [31]) might be silenced and lost following hexaploidization in $A$. sativa. Similar rapid loss of $45 \mathrm{~S}$ rDNA sites is also seen in the tetraploid wheat-relative Aegilops ventri$\cos a$ (with DDNN genome designation), where D-genome 45S rDNA sites are lost [64].

\section{Evolution of repeats \\ Diploid speciation and repetitive DNAs}

The karyotypes with repetitive sequence locations provide a fresh perspective in understanding evolution in Avena. A-genome specific pAs120a was isolated long ago [36]. They discussed the repeat length and existence of four monomers inserted within pAs120a, suggesting cautiously that the pAs120a sequence could be classified as a satellite DNA sequence. In contrast, our sequence clusters homologous to pAs120a showed high similarity to Ogre/Tat and chromovirus retrotransposons (Fig. 2k) indicating that this repeat originated from a retroelement, and might have been generated as a tandem repeat from a retroelement subregion by rolling circle amplification. However, twenty years later, we still share the uncertainty of Linares et al. [36] about the evolution of this sequence to become Agenome-specific. Other sequence families also show differential amplification or reduction in individual Avena A-genomes (Fig. 6a), and in the hexaploid 
compared to the diploid ancestral genomes; e.g. the higher abundance of $\mathrm{C}$-chromosome specific motifs identified in A. sativa genome (e.g. As-T153) or high abundance of D-chromosome specific motifs identified in A. hirtula (Ast-T116; Fig. 6a, Additional file 20: Table S8a).

From molecular dating analyses, the crown age of the $\mathrm{C}$-genome diploid lineage was $\sim 20 \mathrm{Mya}$, older than the crown age of A-genome polyploid lineages [31, 32]. This is supported by greater proportion of $\mathrm{C}$-genome specific motifs, diverging from the common ancestors before the radiation of $\mathrm{A}$ - and $\mathrm{D}$-genome specific motifs, as the $\mathrm{A}$ and D-genome specific motifs amplified independently in common oat (Fig. 6b). This evolutionary scenario is also supported by repeats common to the A- and $\mathrm{D}$-genomes or all three genomes, but no repeats were found to be specific for the C- and A- or C- and D- genomes. Retrotransposons may have a role in genome behaviour by acting as nuclei for RNA-dependent DNA methylation (as [65]), leading to position effect variegation via heterochromatinization around repetitive elements affecting adjacent gene expression [66, 67].

\section{Distal chromosome regions and translocations}

The repetitive sequences used as probes here show major genomic changes through chromosome translocations in common oat [43]. In situ hybridization is unique as a method to show the nature and extend of these translocations, and the use of the repetitive probes (Figs. 2 and 3) isolated here show three kinds of intergenomic translocations:

(a) C-genome segments translocated onto $\mathrm{A}$ - and D-chromosomes - four A-C translocations (pairs 17/ $18 \& 25 / 26$ ) identified by the AF226603_45bp probe (Fig. 4c, Additional file 9: Figure S9a and S9d-S9e), and 12 D-C translocations (chromosomes 29-40) identified by AF226603_45bp probe (Additional file 3: Figure S3a, S3c and S3f, Additional file 5: Figure S5a-S5f);

(b) A- or D-genome segments translocated onto C-chromosomes - four C-A translocations (pairs 5/6 \& 11/12) identified by pAs120a probe (Additional file 4: Figure S4c-S4d, Additional file 5: Figure S5c-S5d); and 10 C-D translocations (chromosomes 1-4, 7-10 \& 13/14) identified by Ast-T116 probe (Fig. $4 \mathrm{~d}-\mathrm{e}$, Additional file 5: Figure S5a-S5c and S5f);

(c) Between A- and D- genome translocations 10 A-D translocations (chromosomes 15/16, 19-24 \& 27/28) identified by Ast-R171 and Ast-T116 probes (Additional file 4: Figure S4b-S4c and S4e-S4f, Additional file 5: Figure S5b-S5c and S5e-S5f), and two D-A translocations (pair 41/42) identified by pAs120a probe (Additional file 5: Figure S5b and S5f, Additional file 7: Figure S7b and S7f).
Translocations can alter chromosome recombination frequencies, and can lead to genetic and evolutionary isolation of new hybrids. The result also suggests the opportunity for introduction of genetic variation as small chromosome segments from wild diploid Avena species into the hexaploid, with recombinant segments involving any genome, or potentially more distant diploid relatives. The oat intergenomic translocations contrast with wheat, where there are no reports of stable intra-genomic translocations with the possible exception of the $4 \mathrm{~A}$ and $4 \mathrm{~B}$ linkage groups, with the $4 \mathrm{~A}$ chromosome differing substantially in repeat content from other chromosomes in the A and B genomes. In oats, the rearranged chromosomes may have adaptive value by enabling different expression levels and modulation of expression of genes from the homoeologous genomes.

\section{Large scale genome organization and implications}

Genomic repeats provide the physical basis for integrating different genomic regions for coordinating interdependent aspects of genome functions [68]. Common oat was inferred to have originated following ancient allotetraploidy (D- and C-genomes) and recent allohexaploidy (ACD-genome) events in the subfamily Pooideae [31]. Given the repeat abundance spanning A- and D-chromosomes, we speculate that the diverged repeats of $A$. strigosa (D-genome) and A. atlantica-A. brevis-A. longiglumis- $A$. wiestii (A-genome) might represent the basis of evolutionary separation of A- and D-genome progenitors, as in other species [69]). Genome-specific repeat amplification, followed by subgenome-function divergence, has been suggested to provide a mechanism driving cold acclimatization in Avena [70], and polyploidization with subgenome dominance may support rust resistance phenotypes that ultimately correspond to agronomic traits [26]. Many evolutionary models suggest that polyploid formation should be associated with a selective advantage, favouring parental genome divergence. Given that six of 13 retrotransposons plus three tandem repeats were more abundant in common oat than in diploid relatives (Additional file 20: Table S8a), it is reasonable to speculate that a burst of ancient repeatassociated genomic duplication may explain expansion of the oat genome size.

\section{Conclusion}

Here, the complete repetitive DNA content of Avena has been surveyed in whole genome data, and the repeats make up $70 \%$ of all the DNA. The most abundant elements were previously described, and we show that a small number of repeat families, some not described before, contribute a high proportion of all the repetitive DNA. It is clear that repeat amplification and turnover of repeat families have been involved during evolutionary separation of the ancestors of common oat, and, in 
combination with frequent intergenomic translocations (not seen in other cereals) and further turnover events, have led to the rapid evolution seen in the hexaploid. Transposable elements are a major contributor to the genome, although the families present, and their relative abundances, differ in Avena from other species groups. Used as a source of DNA markers or chromosomal probes, retroelements have utility in crop breeding [71] and tracking chromosomes in hybrids and translocation lines. With increasing data coming from long-read technologies (including Nanopore, PacBio Sequel and highthroughput chromosome conformation capture), knowledge of the repeat landscape is useful in optimizing the approach to genome sequence assembly by accounting for the abundance and genome distribution of only a small number of repeat families.

\section{Methods}

\section{Plant material}

Eight Avena species (origin of samples is given in Additional file 13: Table S1, chromosome and genome designation see Liu et al. [31], seeds were obtained from CN-Saskatchewan and USDA-Beltsville Germplasm System) were used in this study. The $171.9 \mathrm{~Gb}$ of raw sequences representing $4.58 \times$ to $7.06 \times$ coverage of Avena genomes [A. sativa (66.1 Gb), A. brevis (34.6 $\mathrm{Gb})$, A. hirtula (35.3 Gb), and A. strigosa (35.9 Gb)] were generated by whole-genome shotgun sequencing with $2 \times 250 \mathrm{bp}$ from $500 \mathrm{bp}$ paired-end libraries (Nanjing Genepioneer Biotechnologies Co. Ltd., Illumina HiSeq2500 platform; Additional file 1: Figure S1, Additional file 14: Table S2a). Project data have been deposited at the National Center for Biotechnology Information (NCBI) under BioProject PRJNA407595 (SRR6056489-6056492).

\section{Repeat discovery \\ Graph-based clustering of sequences}

Similarity-based clustering, repeat identification, and classification of a subset paired-end raw reads (1.72-2.87 GB occupied 2.60-8.29\% of Avena genomes; Additional file 14: Table S2b) were performed by RepeatExplorer analysis (Additional file 15: Table S3). It was set with read overlaps containing $\geq 50 \%$ of length with $90 \%$ of similarity as edges to save the potential error of "bridge" reads with partial similarity among two unrelated communities [15]. The longest contigs in each of 821 clusters were analysed by BLAST search against NCBI database to check for repeat identification (Fig. 1, Additional file 16: Table S4) and repetitive DNA composition was summarized manually (Additional file 17: Table S5). Primer pairs were designed from one contig of each retroelement or tandem repeat belonging to clusters of no (or less) 1st-order neighbours [72] (Additional file 12: Figure S12a-S12t, Additional file 18: Table S6, Additional file 19: Table S7), probe designations use genus and species abbreviation plus $\mathrm{T}$ for tandem or $\mathrm{R}$ for retrotransposon type followed by the cluster number. Primers designed from clones pTa71 [34] and pTa794 [35] were used for $45 \mathrm{~S}$ and $5 \mathrm{~S}$ rDNA amplification respectively (Additional file 18: Table S6). Cluster graphs, dotplots and FISH probe copy numbers were investigated by SeqGrapheR v.3.3.1 [15] and Geneious [38] (Fig. 2a-p, Additional file 20: Table S8).

\section{k-mer analysis}

Genome repetitivity was assessed via 10- to 64-mer frequencies by the program Jellyfish v.2.2.6 [14] for four Avena species (Fig. 3a-e). Frequencies were compared with Petunia axillaris, potato, sorghum, and tomato [21, 39-41] (Fig. 3f). The As_16mer43bp consensus sequence was used to design a synthetic oligonucleotide probe (Additional file 18: Table S6), its position was aligned to RepeatExplorer cluster graph layouts (Fig. 2a).

\section{Multicolour fluorescence in situ hybridization (FISH)}

Root tips were fixed in 96\% ethanol: glacial acetic acid (3:1) for at least $1.5 \mathrm{~h}$ and stored in the fixative at $-20^{\circ}$ $\mathrm{C}$ overnight. An enzyme solution with $0.2 \%$ Cellulase Onozuka R10 (Yakult Pharmaceutical, Tokyo), 2\% Cellulase (C1184 Sigma-Aldrich) and 3\% Pectinase (P4716; Sigma-Aldrich, St Louis, USA) was used to digest root tips for $90 \mathrm{~min}$ at $37^{\circ} \mathrm{C}$. Root tips were macerated in a drop of $60 \%$ acetic acid, and roots were squashed gently under a coverslip.

PCR amplification was performed in $25 \mu \mathrm{l}$ reaction mixture containing 50-100 ng genomic DNA, $0.2 \mu \mathrm{M}$ each primer, $0.4 \mathrm{mM}$ deoxynucleotide triphosphate mix, $1 \times$ PCR

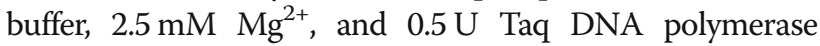
(Kapa Biosystems). PCR conditions were $95^{\circ} \mathrm{C}$ for $3 \mathrm{~min}$, followed by 35 cycles of $95^{\circ} \mathrm{C}$ for $30 \mathrm{~s}, 51.3-68.0^{\circ} \mathrm{C}$ for $30 \mathrm{~s}$, and $72^{\circ} \mathrm{C}$ for $1 \mathrm{~min}$. PCR products were electrophoresed on 1.0\% agarose gels, purified using an E.Z.N.A. Cycle Pure Kit (Omega), and then labelled with either biotin-16-dUTP or digoxigenin-11-dUTP (Roche Diagnostics) using a BioPrime Array Comparative genomic hybridization (CGH) Genomic Labelling System (Invitrogen).

FISH was performed as described by Schwarzacher and Heslop-Harrison [73]. The $34 \mu \mathrm{l}$ of hybridization mixture (stringency $76 \%$ ), containing $50 \%$ formamide, $2 \times$ SSC (Saline Sodium Citrate buffer; $0.3 \mathrm{M} \mathrm{NaCl}, 0.03 \mathrm{M}$ sodium citrate) $10 \%$ dextran sulphate, $0.125 \%$ SDS (sodium dodecyl sulphate), $0.125 \mathrm{mM}$ EDTA (ethylenediamine-tetraacetic acid); $1 \mu \mathrm{g}$ sheared salmon sperm DNA and up to $1000 \mathrm{ng}$ of labelled probes were applied on each slide. After 
denaturation at $72{ }^{\circ} \mathrm{C}$ for $7 \mathrm{~min}$, hybridization at $37^{\circ} \mathrm{C}$ overnight in a ThermoHybaid HyPro-20, slides were washed in $0.1 \times \mathrm{SSC}$ at $42^{\circ} \mathrm{C}$, . FISH probe hybridization sites were detected via fluorescein isothiocyanate (FITC) conjugated anti-digoxigenin $(200 \mu \mathrm{g} / \mathrm{ml}$; Roche Diagnostics), Streptavidin Alexa Fluor 594 $(200 \mu \mathrm{g} / \mathrm{ml}$; Molecular Probes) or Streptavidin Alexa Fluor 647 $(1 \mathrm{mg} / \mathrm{ml}$; Molecular Probes) in $4 \times$ SSC, $0.1 \%$ Tween 20, and 5\% BSA (Bovine Serum Albumin). Slides were counterstained with 4', 6-diamidino-2-phenylindole (DAPI; $3 \mathrm{mg} / \mathrm{ml}$ )-antifade solution (AF1, Citifluor, London, UK; 50\%). FISH images were captured by a Nikon Eclipse 80i epifluorescent microscope fitted with appropriate sets of $\mathrm{t}$ band-pass filters to capture Alexa 647, Alexa 594, Tetrachlorofluorescein (TET), FITC and DAPI, a DS-QiMc monochromatic camera, and NIS-Elements v.2.34 (Nikon, Tokyo, Japan). For each metaphase, four single channel images (pseudocoloured, yellow, red, green, and blue respectively) of $1280 \times 1024$ pixel size were analysed by Image J v.1.51j8 (Wayne Rasband, NIH, USA) and superimposed in Photoshop CS6 v.13.0 (Adobe System, San Jose, CA, USA). Total 269 slides including 2353 metaphases of 8 species (average 8 metaphases per slide) were observed for in situ hybridization analyses. Probe fragment copy numbers were determined by counting the number of reads (per genome equivalent) mapping to the probe fragment sequence ("Map to Reference").

\section{Additional files}

Additional file 1: Figure S1. Spikelet morphology of eight sampled Avena species. a A. atlantica: the dispersion units-the upper florets are attached to the lower floret and only the lower floret show disarticulation. b A. brevis: spikelets show persistent florets with bidenticulate lemma tips at maturity. c A. hirtula: a Mediterranean wild type with lemma bristles 6-10 $\mathrm{mm}$. d A. longiglumis: 2-3 florets/ spikelet, each floret is disarticulated; lemma back is covered with dense hairs. e A. strigosa: 2-3 florets/spikelet and persistent florets. f A. wiestii: desert and steppe wild type with lemma bristles $5-8 \mathrm{~mm} . \mathrm{g}$ A. eriantha: glumes markedly unequal in size. $\mathrm{h}$ A. sativa: spikelets $1.5-4 \mathrm{~cm}$ with typically spread glumes at maturity. Scale bars $=1 \mathrm{~cm}$. ( $\mathrm{TIF} 4210 \mathrm{~kb}$ )

Additional file 2: Figure S2. Distribution of graph-based clusters. Hierarchical agglomeration of RepeatExplorer analyses of four Avena species genomes are shown. a A. sativa S312. b A. brevis B289. C A. hirtula. H299. d A. strigosa S135. Coloured bars denote clusters $\geq 0.01 \%$ of genome: $x$-axis denotes the cumulative read number percentage while $y$-axis denotes the read numbers in the clusters. Bars coloured according to the repeat types of cluster annotation (Additional files 15: Table S3). (TIF $1895 \mathrm{~kb}$ )

Additional file 3: Figure S3. Localization of selected C-genome specific repetitive sequences on Avena sativa metaphase chromosomes by multicolour FISH. Probe signals were captured individually with a black and white CCD camera and then pseudo-coloured to create overlaid images. For detailed description of signal distribution see Additional file 21: Table S9. a AF226603_45bp (hybridization sites displayed in red), Ab-R18 amplified from A. atlantica (green), and pAs120a from A. atlantica (blue). Note that overlapping signals of the red and green probe give yellow signals. b Ab-R19 (red) and Ab-R126 (green), counterstain DAPI (blue) shown on all chromosomes. Note that overlapping signals of the red and green probe appear white, but show several chromosome ends not labelled by either probe appearing blue or show green double dots. $c$ AF226603_45bp (red), Ah-T118 from A. hirtula (green), and pAs120a from A. hirtula (blue). Overlapping signals of the red and green probe appear yellow and show non-uniform labelling of chromosomes. An interphase nucleus is visible at the bottom of the image. d As-T119 (green), double-dots (starred) appearing in yellow on top of the red signal of As_16mer43bp. DAPI fluorescence shown in blue.e As-T175 (green, doubledots) and As_16mer43bp (red) showing large blocks of hybridization signal on C genome chromosomes (starred). DAPI fluorescence shown in blue. $f$ TET labeled AF226603_45bp (red), As_16mer43bp (pink), and DAPI (blue). Scale bars $=5 \mu \mathrm{m}$. (TIF $6648 \mathrm{~kb}$ )

Additional file 4: Figure S4. Fluorescent in situ hybridization (FISH) karyotyping of Avena sativa. Probes are AF226603_45bp (direct TET, red) for the C genome, pAs120a (biotin, Alexa 594, blue) for the A genome, and Ast-R171 (digoxigenin, FITC, green) for the D genome. On the right the DAPI image of chromosomes is shown in white, in the middle the same metaphase shows hybridization signal from all three probes except in (d) where only AF226603_45bp in yellow and pAs120 in blue are visible. Probes were amplified from different diploid species. a A. brevis. b A. atlantica. c A. strigosa. d A. wiestii. e A. longiglumis. f A. hirtula. In the karyotypes (on the left), chromosomes are arranged in rows corresponding to genome origin: 1-14 C-genome, 15-28 A-genome, and 29-42 D-genome. White stars, arrows, and arrowheads denoted C-, A-, and D-chromosome regions, translocated to a different genome: there are $C$ translocations on 12 D-chromosomes (29-40); A translocations on four C-chromosomes (5/6 \& 11/12); D translocations on 10 A-chromosomes $(15 / 16,19-24 \& 27 / 28)$. Scale bars $=5 \mu \mathrm{m}$. (TIF $7816 \mathrm{~kb})$

Additional file 5: Figure S5. Fluorescent in situ hybridization (FISH) karyotyping of Avena sativa. Probes are AF226603_45bp (direct TET, red) for the C genome, pAs120a (biotin, Alexa 594; blue) for the A genome, and Ast-T116 (digoxigenin, FITC, green) for the D genome. On the right the DAPI image of chromosomes is shown in white, in the middle the same metaphase shows hybridization signal from all three probes except in (d) and (e) where only AF226603_45bp in yellow and pAs120 in blue are visible. Probes were amplified from different diploid species. a $A$. brevis. b A. atlantica. c A. strigosa. d A. wiestii. e A. longiglumis. f A. hirtula. In the karyotypes (on the left), chromosomes are arranged in rows corresponding to genome origin: 1-14 C-genome, 15-28 A-genome, and 29-42 D-genome. White circles denote nucleolus organizer regions (NORs) signals. White stars, arrows, and arrowheads denoted $C_{-}, A_{-}$-, and D-chromosome regions, translocated to a different genome: there are $D$ translocations on ten C-chromosomes (1-4, 7-10 \& 13/14); there are C translocations on $10 \mathrm{D}$-chromosomes (29-40); A translocations on four C-chromosomes (5/6 \& 11/12); D translocations on 10 A-chromosomes $(15 / 16,19-24 \& 27 / 28)$. Scale bars $=5 \mu \mathrm{m}$. (TIF $8170 \mathrm{~kb}$ )

Additional file 6: Figure S6. Fluorescent in situ hybridization (FISH) of Avena sativa. Single channel images of far-red $(a, d, g)$, pseudocoloured blue $(b, e, h)$, and green $(c, f, i)$ dotted lines circling C, A and D-genome chromosomes analysed by Image J V.1.51j8 for Figs. 4a (a-c), 4e (d-f) and 5c (g-i). (a-c) FISH probes AF226603_45bp (direct TET, red), pAs120a (biotin, Alexa 594; blue) and Ast-R171 (digoxigenin, FITC, green) and from A. strigosa: separation of the overlapped chromosomes (a) 6, (b) 21 \& 25, 16, 23 \& 24, and (c) 29 \& 42 and 31 \& 41 for Fig. 4d. (d-f) FISH probes AF226603_45bp (far red), Ast-T116 (green) and pAs120a (pseudocoloured blue) from A. strigosa: separation of the overlapped chromosomes (d) 3, 6, 12 \& 14, (e) 18 \& 27, and (f) 33 \& 42 for Fig. 4e. (g-i) AF226603_45bp (far red), A/D-genome specific Ab-T166 (green), and pAs120a (pseudocoloured blue) from $A$. atlantica: separation of the overlapped chromosomes (g) 7, 8 \& 14, (h) 18, 23, 24, 25 \& 26, and (i) 31 \& 36 for Fig. 5c. (TIF 6215 kb)

Additional file 7: Figure S7. Fluorescent in situ hybridization (FISH) karyotyping of Avena sativa. Probes are AF226603_45bp (direct TET, red) for the C genome, pAs120a (biotin, Alexa 594; blue) for the A genome, and Ab-R126 (digoxigenin, FITC, green). On the right the DAPI image of chromosomes is shown in white, in the middle the same metaphase shows hybridization signal. Probes were amplified from different diploid species. a A. brevis. b A. atlantica. c A. strigosa. 
d A. wiestii. e A. longiglumis. f A. hirtula. In the karyotypes (on the left), chromosomes are arranged in rows corresponding to genome origin: 1-14 C-genome, 15-28 A-genome, and 29-42 D-genome. White stars, arrows, and arrowheads denoted $C_{-}, A_{-}$, and D-chromosome regions, translocated to a different genome. Scale bars $=5 \mu \mathrm{m}$. (TIF $8552 \mathrm{~kb}$ )

Additional file 8: Figure S8. Fluorescent in situ hybridization (FISH) karyotyping of Avena sativa. Probes are AF226603_45bp (direct TET, red) for the C genome, pAs120a (biotin, Alexa 594; blue) for the A genome, and Ah-R31 (digoxigenin, FITC, green). On the right the DAPI image of chromosomes is shown in white, in the middle the same metaphase shows hybridization signal. Probes were amplified from different diploid species. a A. brevis. b A. atlantica. c A. strigosa. d A. wiestii. e A. longiglumis. f $A$. hirtula. In the karyotypes (on the left), chromosomes are arranged in rows corresponding to genome origin: 1-14 C-genome, 15-28 A-genome, and 29-42 D-genome. White stars, arrows, and arrowheads denoted C-, A-, and Dchromosome regions, translocated to a different genome. Additionally, white diamonds denote strong green band signal in Additional file 8: Figure S8e). Scale bars $=5 \mu \mathrm{m}$. (TIF $7444 \mathrm{~kb}$ )

Additional file 9: Figure S9. Fluorescent in situ hybridization (FISH) karyotyping of Avena sativa. Probes are AF226603_45bp (direct TET, red) for the C genome, pAs120a (biotin, Alexa 594; blue) for the A genome, and Ab-T148 (digoxigenin, FITC, green). On the right the DAPI image of chromosomes is shown in white, in the middle the same metaphase shows hybridization signal. Probes were amplified from different diploid species. a A. brevis. b A. atlantica. c A. strigosa. d A. wiestii. e A. longiglumis. f A. hirtula. In the karyotypes (on the left), chromosomes are arranged in rows corresponding to genome origin: 1-14 C-genome, 15-28 A-genome, and 29-42 Dgenome. White stars, arrows, and arrowheads denoted C-, A-, and D-chromosome regions, translocated to a different genome. Additionally, white diamonds denote strong green band signal in Additional file 9: Figure S9c-e). Scale bars $=5 \mu \mathrm{m}$. (TIF $8041 \mathrm{~kb}$ )

Additional file 10: Figure S10. Fluorescent in situ hybridization (FISH) of Avena sativa. Chromosomal distribution of biotin labelled As_16mer43bp (red) and selected fluorescence in situ hybridization (FISH) probes labelling C-, A- and D-genome chromosomes of common oat (Avena sativa). a Digoxigenin labeled Ah-R52 (green) from A. hirtula. b Digoxigenin labeled As-R133 (green) from A. longiglumis. c Digoxigenin labeled Ast-R155 (green) from A. wiestii. d Digoxigenin labeled Ast-R176 (green) from A. hirtula. e Digoxigenin labeled Ah-T125 (green) from A. wiestii. f Digoxigenin labeled Ast-T125 (green) from A. wiestii. Scale bars $=5 \mu \mathrm{m}$. (TIF $8865 \mathrm{~kb}$ )

Additional file 11: Figure S11. rDNA sequence assembly and FISH. (a-b) Alignment of cluster contig sequences to assemble the $45 \mathrm{~S}$ (a) and $5 S$ rDNA (b) of Avena sativa. (c-e) FISH karyotyping of A. sativa with AF226603_45bp (direct TET, red), rDNA, and others. On the right the DAPI image of chromosomes is shown in white, in the middle the same metaphase shows hybridization signal. In the karyotypes (on the left), chromosomes are arranged in rows corresponding to genome origin: 1-14 C-genome, 15-28 A-genome, and 29-42 D-genome. White angle brackets and pigeons denoted $45 \mathrm{~S}$ and $5 \mathrm{~S}$ rDNA signals, respectively. $\mathrm{C}$ Digoxigenin labeled Ab-T148 (green) from A. wiestii and biotin labeled pTa71_45S (blue). d Digoxigenin labeled Ab-T148 (green) from A. wiestii and biotin labeled pTa794_5S (blue). e Digoxigenin labeled pTa794_5S (green) and biotin labeled pTa71_45S (blue) from T. aestivum. Scale bars $=5 \mu \mathrm{m}$. (TIF $4200 \mathrm{~kb}$ )

Additional file 12: Figure S12. Analysis of neighboring RepeatExplorer clusters. The interactive graphs showing selected clusters harboring FISH probes used in the present study (red circles) with 1st order neighbors with at least one connection (green circles). Connection between two neighboring clusters shown by grey and black lines along with similar read number. a 312CL82. b 312CL83. c 312CL119. d 312CL125. e 312CL133. f 312CL151. g 312CL153. h 289CL18. i 289CL19. j 289CL93. k 289CL105. I 289CL126. m 289CL145. n 289CL159. o 289CL166. p 299CL31. q 299CL52. r 315CL87. s 315CL116. t 315CL125. Clusters with at least one neighbor shown in circle. Remaining clusters 289CL148, 289CL187, 299CL118, 299CL125, 299CL126, 312CL175, 315CL155, 315CL171 and 315CL176 without any one neighbor. Black letters denote tandem repeats (b, $c, f-g, j-k, m-o, s-t)$ and brown-red letters denote linear cluster graphs of non-tandem repeats (a, d-e, h-i, l, p-r). (JPG 457 kb)

Additional file 13: Table S1. Material used in this study. Source and origin, species name, authority, chromosome number and genome designation are given for the eight Avena samples used in this study. Repeat Cluster and spikelet figures are also listed. (DOCX $27 \mathrm{~kb}$ )

Additional file 14: Table S2. Statistics of whole-genome shotgun sequencing reads and RepeatExplorer analyses of Avena species. Haploid genome size and whole-genome shotgun sequencing reads statistics of four Avena species are given in (a) and RepeatExplorer summary data in (b). (DOCX $17 \mathrm{~kb}$ )

Additional file 15: Table S3. RepeatExplorer analyses of four Avena species. Statistics of cluster, read number and genome proportion of repetitive DNA composition (a) and the cluster (greater than or equal to $1.00 \%$ of genome) annotation by RepeatExplorer analyses (b) of four Avena species are given. (DOCX $40 \mathrm{~kb}$ )

Additional file 16: Table S4. The NCBI blast results of 821 clusters of four Avena species. Cluster and the longest contigs within, description, query coverage and identity are listed for each cluster. (DOCX $92 \mathrm{~kb}$ )

Additional file 17: Table S5. Repetitive DNA composition of genomes of four Avena species. Genome portion (as percent) is listed for each species. Families and subfamilies of Transposable elements Class I (retrotransposons) and Class II (DNA transposons), and Tandem repeats are also given. (DOCX $22 \mathrm{~kb})$

Additional file 18: Table S6. Oligonucleotides used to generate FISH probes. Primer pairs, annealing temperatures, the expected fragment sizes and lables for repeats identified from k-mer or RepeatExplorer analyses of four Avena species. (DOCX $23 \mathrm{~kb}$ )

Additional file 19: Table S7. RepeatExplorer characterization of selected repeat clusters of four Avena species. Characterization of repetitive fragments used as FISH probes from k-mer or RepeatExplorer analyses of Avena species. Genome proportion, domain hits, contig length and FISH figure references are listed. (DOCX 24 kb)

Additional file 20: Table S8. Genomic copy number and relative proportion of selected repeats of Avena species. PairedEnd fasta data were used to for "Map to Reference" tool in Geneious v.10.0.7 (Kearse et al., 2012). (DOCX $25 \mathrm{~kb}$ )

Additional file 21: Table S9. FISH signal distribution patterns on Avena sativa chromosomes using probes for selected repeats. Descriptions are based on analysis of 269 slides and 2353 metaphase (avg. 8 metaphases per slide) were analysed and chromosomes assigned to genomes C, A or D according to Sanz et al. (2010). Ten probes are $C$ genome specific, one probe A genome specific, two probe D genome specific, five probes A and $\mathrm{D}$ genome specific and seven probes hybridized to all three genomes. (DOCX $25 \mathrm{~kb}$ )

Additional file 22: Table S10. The NCBI blast results of tandem repeats in Fig. 6b. Genome specificity, satellite family designation, monomer lengths and $\mathrm{NCBI}$ accession of twelve tandem repeats are listed. (DOCX $28 \mathrm{~kb})$

\section{Abbreviations}

Ab: Avena brevis; Ah: Avena hirtula; As: Avena sativa; Ast: Avena strigosa; BLAST: Basic local alignment search tool; BSA: Bovine serum albumin; CGH: Comparative genomic hybridization; DAPI: 4', 6-diamidino-2-phenylindole; EDTA: Ethylenediamine-tetraacetic acid; EnSpm: Enhancer suppressor mutator; FISH: Fluorescent in situ hybridization; FITC: Fluorescein isothiocyanate; GC: Guanine-cytosine; INT: Integrase; LINE: Long interspersed nuclear element; LTR: Long terminal repeat; NCBI: National Center for Biotechnology Information; NOR: nucleolus organizer region; PCR: Polymerase chain reaction; rDNA: Ribosomal DNA; RH: RnaseH; RT: Reverse transcriptase; satDNA: Satellite DNA; SDS: Sodium dodecyl sulphate; SINEs: Short interspersed nuclear element; SSC: Saline sodium citrate; TE: Transposable element; TET: Tetrachlorofluorescein; WGD: Whole genome duplication 


\section{Acknowledgements}

We thank CN-Saskatchewan and USDA-Beltsville Germplasm System for seeds, ELIXIR-CZ project (LM2015047) for Galaxy based RepeatExplorer resources, National Supercomputing Center in Guangzhou for data analyses, Dr. J. Bailey for help with chromosome preparations, and Dr. R.H.M. Salih for assistance with microscopy.

\section{Funding}

This work was funded by Overseas Distinguished Scholar Project of SCBG to J.S. Heslop-Harrison (Y861041001), Undergraduate Innovation Training Program of Chinese Academy of Sciences (59), Strategic Priority Research Program of Chinese Academy of Science (XDA13020602), and Special Basic Research Foundation of Ministry of Science and Technology of People's Republic of China (2013FY112100). The funders had no role in study design, data collection and analysis, decision to publish, or preparation of the manuscript.

\section{Availability of data and materials}

All the data pertaining to the present study have been included in table and/or figure form in the manuscript and authors are pleased to share analyzed/raw data and plant materials upon reasonable request. Other datasets supporting the conclusions of this article are included within the article and its additional files.

\section{Authors' contributions}

QL, TS, JSHH designed the experiments; QL, XYL, XYZ, MZL, FJZ performed the probes design, FISH and analyzed the data, QL, TS, JSHH wrote the manuscript. All authors have read and approved the manuscript.

\section{Ethics approval and consent to participate}

Not applicable.

\section{Consent for publication}

Not applicable.

\section{Competing interests}

The authors have declared that no competing interests exist.

\section{Publisher's Note}

Springer Nature remains neutral with regard to jurisdictional claims in published maps and institutional affiliations.

\begin{abstract}
Author details
${ }^{1}$ Key Laboratory of Plant Resources Conservation and Sustainable Utilization / Guangdong Provincial Key Laboratory of Applied Botany, South China Botanical Garden, Chinese Academy of Sciences, Guangzhou, China. ${ }^{2}$ University of Chinese Academy of Sciences, Beijing, China. ${ }^{3}$ Genepioneer Biotechnologies Co. Ltd., Nanjing, China. ${ }^{4}$ Institute of Botany, Jiangsu Province and Chinese Academy of Sciences, Nanjing, China. ${ }^{5}$ Department of Genetics and Genome Biology, University of Leicester, Leicester LE1 7RH, UK.
\end{abstract}

\section{Received: 26 December 2018 Accepted: 9 April 2019}

\section{Published online: 30 May 2019}

\section{References}

1. Heslop-Harrison JS, Schwarzacher T. Organization of the plant genome in chromosomes. Plant J. 2011;66(1):18-33.

2. Ruprecht C, Lohaus R, Vanneste K, Mutwil M, Nikoloski Z, Van de Peer Y, et al. Revisiting ancestral polyploidy in plants. Sci Adv. 2017;3(7):e1603195.

3. Vakirlis N, Sarilar V, Drillon G, Fleiss A, Agier N, Meyniel JP, et al. Reconstruction of ancestral chromosome architecture and gene repertoire reveals principles of genome evolution in a model yeast genus. Genome Res. 2016;26(7):918-32.

4. O'Connor RE, Fonseka G, Frodsham R, Archibald AL, Lawrie M, Walling GA, et al. Isolation of subtelomeric sequences of porcine chromosomes for translocation screening reveals errors in the pig genome assembly. Animal Genet. 2017;48(4):395-403.

5. Mandáková T, Li Z, Barker MS, Lysak MA. Diverse genome organization following 13 independent mesopolyploid events in Brassicaceae contrasts with convergent patterns of gene retention. Plant J. 2017;91(1):3-21.
6. De Koning APJ, Gu W, Castoe TA, Batzer MA, Pollock DD. Repetitive elements may comprise over two-thirds of the human genome. PLoS Genet. 2011;7(12):e1002384.

7. Biscotti MA, Olmo E, Heslop-Harrison JS. Repetitive DNA in eukaryotic genomes. Chromosom Res. 2015;23(3):415-20.

8. Maumus F, Quesneville $\mathrm{H}$. Impact and insights from ancient repetitive elements in plant genomes. Curr Opin Plant Biol. 2016;30(2):41-6.

9. Treangen TJ, Salzberg SL. Repetitive DNA and next-generation sequencing: computational challenges and solutions. Nat Rev Genet. 2012;13(1):36-46.

10. Nazaryan-Petersen L, Eisfeldt J, Pettersson M, Lundin J, Nilsson D, Wincent J, et al. Replicative and non-replicative mechanisms in the formation of clustered CNVs are indicated by whole genome characterization. PLoS Genet. 2018;14(11):e1007780

11. Novák P, Neumann P, Pech J, Steinhaisl J, Macas J. RepeatExplorer: a galaxy-based web server for genome-wide characterization of eukaryotic repetitive elements from next-generation sequence reads. Bioinformatics. 2013;29(6):792-3.

12. Bauer E, Schmutzer T, Barilar I, Mascher M, Gundlach H, Martis MM, et al. Towards a whole-genome sequence for rye (Secale cereale L.). Plant J. 2017; 89(5):853-69.

13. Gaiero P, Vaio M, Peters SA, Schranz ME, de Jong H, Speranza PR. Comparative analysis of repetitive sequences among species from the potato and the tomato clades. Ann Bot. 2019;123(3):521-32.

14. Marçais G, Kingsford C. A fast, lock-free approach for efficient parallel counting of occurrences of k-mers. Bioinformatics. 2011;27(6):764-70.

15. Novák P, Neumann P, Macas J. Graph-based clustering and characterization of repetitive sequences in next-generation sequencing data. BMC Bioinformatics. 2010;11(1):378.

16. Heslop-Harrison JS, Schwarzacher T. Nucleosomes and centromeric DNA packaging. Proc Natl Acad Sci U S A. 2013;150(50):19974-5.

17. Maças J, Neumann P, Navrátilová A. Repetitive DNA in the pea (Pisum sativum L.) genome: comprehensive characterization using 454 sequencing and comparison to soybean and Medicago truncatula. BMC Genomics. 2007;8(1):427.

18. Hřibová E, Neumann P, Matsumoto T, Roux N, Macas J, Doležel J. Repetitive part of the banana (Musa acuminata) genome investigated by low-depth 454 sequencing. BMC Plant Biol. 2010;10:204.

19. Renny-Byfield S, Chester M, Kovarík A, le Comber SC, Grandbastien MA, Deloger $\mathrm{M}$, et al. Next generation sequencing reveals genome downsizing in allotetraploid Nicotiana tabacum, predominantly through the elimination of paternally derived repetitive DNAs. Mol Biol Evol. 2011;28(10):2843-54.

20. He QY, Cai ZH, Liu HJ, Bao CL, Mao WH, Jin WW. Repetitive sequence and karyotyping reveals centromere-associated DNA sequences in radish (Raphanus sativus L.). BMC Plant Biol. 2015;15:105.

21. Bombarely A, Moser M, Amrad A, Bao MZ, Bapaume L, Barry CS, et al. Insight into the evolution of the Solanaceae from the parental genomes of Petunia hybrida. Nat Plants. 2016;2(6):16074.

22. Andon MB, Anderson JW. The oatmeal-cholesterol connection: 10 years later. Am J Lifestyle Med. 2008;2(1):51-7.

23. EFSA Panel on Dietetic Products and Nutrition and Allergies (EFSA). Scientific opinion on the substantiation of a health claim related to oat beta-glucan and lowering blood cholesterol and reduced risk of (coronary) heart disease pursuant to article 14 of regulation (EC) no 1924/2006. EFSA J. 2010;8(12):1885.

24. Maças J, Neumann P, Novák P, Jiang JM. Global sequence characterization of rice centromeric satellite based on oligomer frequency analysis in largescale sequencing data. Bioinformatics. 2010;26(17):2101-8.

25. Luo MC, Gu YQ, Puiu D, Wang H, Twardziok SO, Deal KR, et al. Genome sequence of the progenitor of the wheat $D$ genome Aegilops tauschii. Nature. 2017;551(7681):498-502.

26. Oliver RE, Tinker NA, Lazo GR, Chao SM, Jellen EN, Carson ML, et al. SNP discovery and chromosome anchoring provide the first physically-anchored hexaploid oat map and reveal synteny with model species. PLoS One. 2013; 8(3):e58068.

27. Song GY, Huo PJ, Wu B, Zhang ZW. A genetic linkage map of hexaploid naked oat constructed with SSR markers. Crop J. 2015;3(4):353-7.

28. Yan HH, Martin SL, Bekele WA, Latta RG, Diederichsen A, Peng YY, et al. Genome size variation in the genus Avena. Genome. 2016;59(3):209-20.

29. Jellen EN, Gill BS, Cox TS. Genomic in situ hybridization differentiates between a/D- and C-genome chromatin and detects intergenomic translocations in polyploid oat species (genus Avena). Genome. 1994; 37(4):613-8. 
30. Katsiotis A, Loukas M, Heslop-Harrison JS. Repetitive DNA, genome and species relationships in Avena and Arrhenatherum (Poaceae). Ann Bot. 2000; 86(6):1135-42

31. Liu Q, Lin L, Zhou XY, Peterson PM, Wen J. Unraveling the evolutionary dynamics of ancient and recent polyploidization events in Avena (Poaceae). Sci Rep. 2017:7(1):41944.

32. Fu YB. Oat evolution revealed in the maternal lineages of 25 Avena species. Sci Rep. 2018;8(1):4252

33. Zhou XY. The A-genome origin of Avena sativa defined by major repetitive DNA classes. M.Sc. Thesis, Guangzhou: South China botanical garden, Chinese Academy of Science; 2018. p. 131.

34. Gerlach WL, Bedbrook JR. Cloning and characterization of ribosomal RNA genes from wheat and barley. Nucl Acids Res. 1979;7(7):1869-85.

35. Gerlach WL, Dyer TA. Sequence organization of the repeating units in the nucleus of wheat which contain 55 rRNA genes. Nucl Acids Res. 1980; 8(21):4851-65

36. Linares $C$, Ferrer E, Fominaya A. Discrimination of the closely related a and $D$ genomes of the hexaploid oat Avena sativa L. Proc Natl Acad Sci U S A. 1998;95(21):12450-5.

37. Ananiev EV, Vales MI, Phillips RL, Rines HW. Isolation of a/D and C genome specific dispersed and clustered repetitive DNA sequences from Avena sativa. Genome. 2002:45(2):431-41.

38. Kearse M, Moir R, Wilson A, Stones-Havas S, Cheung M, Sturrock S, et al. Geneious basic: an integrated and extendable desktop software platform for the organization and analysis of sequence data. Bioinformatics. 2012;28(12):1647-9.

39. Paterson AH, Bowers JE, Bruggmann R, Dubchak I, Grimwood J, Gundlach H, et al. The Sorghum bicolor genome and the diversification of grasses. Nature. 2009;457(7229):551-6.

40. Xu X, Pan S, Cheng SF, Zhang B, Mu DS, Ni PX, et al. Genome sequence and analysis of the tuber crop potato. Nature. 2011;475(7355):189-95.

41. Tomato Genome Consortium (TGC). The tomato genome sequence provides insights into fleshy fruit evolution. Nature. 2012;485(7400):635-41.

42. Bennetzen $J$, Wang $H$. The contributions of transposable elements to the structure, function, and evolution of plant genomics. Annu Rev Plant Biol. 2014;65(1):505-30.

43. Sanz $M$, Jellen $E$, Loarce $Y$, Irigoyen $M$, Ferrer $E$, Fominaya A. A new chromosome nomenclature system for oat (Avena sativa L. and A. byzantina C. Koch) based on FISH analysis of monosomic lines. Theor Appl Genet. 2010;121(8):1541-52

44. Katsiotis A, Schmidt T, Heslop-Harrison JS. Chromosomal and genomic organisation of Ty1-copia-like retrotransposon sequences in the genus Avena. Genome. 1996;39(2):410-7.

45. Wang GX, He QY, Macas J, Novák P, Neumann P, Meng DX, et al. Karyotypes and distribution of tandem repeat sequences in Brassica nigra determined by fluorescence in situ hybridization. Cytogenet Genome Res. 2017;152(3):158-65.

46. Wicker T, Schulman AH, Tanskanen J, Spannagl M, Twardziok S, Mascher M, et al. The repetitive landscape of the $5100 \mathrm{Mbp}$ barley genome. Mob DNA. 2017;8:22.

47. Kuhn GCS, Küttler H, Moreira-Filho O, Heslop-Harrison JS. The 1.688 repetitive DNA of Drosophila: concerted evolution at different genomic scales and association with genes. Mol Biol Evol. 2011;29(1):7-11.

48. International Rice Genome Sequencing Project. The map-based sequence of the rice genome. Nature. 2005;436(7052):793-800.

49. Schnable PS, Ware D, Fulton RS, Stein JC, Pasternak S, Liang C, et al. The B73 maize genome: complexity, diversity, and dynamics. Science. 2009; 326(5956):1112-5.

50. International Brachypodium Initiative. Genome sequencing and analysis of the model grass Brachypodium distachyon. Nature. 2010;463(7282):763.

51. The International Wheat Genome Sequencing Consortium (IWGSC). Shifting the limits in wheat research and breeding using a fully annotated reference genome. Science. 2018;361(6403):aar7191.

52. Cai ZX, Liu HJ, He QY, Pu MW, Chen J, Lai JS, et al. Differential genome evolution and speciation of Coix lacryma-jobi L. and Coix aquatica Roxb. Hybrid guangxi revealed by repetitive sequence analysis of fine karyotyping. BMC Genomics. 2014;15(1):1025-40.

53. Belser C, Istace B, Denis E, Dubarry M, Baurens FC, Falentin C, et al. Chromosome-scale assemblies of plant genomes using nanopore long reads and optical maps. Nat Plants. 2018;4(11):879-87.

54. Schorn AJ, Gutbrod MJ, LeBlanc C, Martienssen R. LTR-Retrotransposon control by tRNA-derived small RNAs. Cell. 2017;170(1):61-71.
55. Richert-Pöggeler KR, Noreen F, Schwarzacher T, Harper G, Hohn T. Induction of infectious petunia vein clearing (pararetro) virus from endogenous provirus in petunia. EMBO J. 2003;22(18):4836-45.

56. Lyu H, He Z, Wu Cl, Shi S. Convergent adaptive evolution in marginal environments: unloading transposable elements as a common strategy among mangrove genomes. New Phytol. 2018;217(1):428-38.

57. Antony Pass D, Sornay E, Marchbank A, Crawford MR, Paszkiewicz K, Kent NA, et al. Genome-wide chromatin mapping with size resolution reveals a dynamic subnucleosomal landscape in Arabidopsis. PLoS Genet. 2017;13(9):e1006988.

58. Vershinin AV, Heslop-Harrison JS. Comparative analysis of the nucleosomal structure of rye, wheat and their relatives. Plant Mol Biol. 1998;36(1):149-61.

59. Kaplan N, Moore IK, Fondufe-Mittendorf Y, Gossett AJ, Tillo D, Field Y, et al. The DNA-encoded nucleosome organization of a eukaryotic genome. Nature. 2009;458(7236):362-6.

60. Alix K, Joets J, Dunsdon CD, Heslop-Harrison P. The CACTA transposon Bot1 played a major role in Brassica genome divergence and gene proliferation. Plant J. 2008;56(6):1030-44.

61. Li CD, Rossnagel BG, Scoles GJ. Tracing the phylogeny of the hexaploid oat Avena sativa with satellite DNAs. Crop Sci. 2000;40(6):1755-63.

62. Niwa O, Kominami R. Untargeted mutation of the maternally derived mouse hypervariable minisatellite allele in F1 mice born to irradiated spermatozoa. Proc Natl Acad Sci U S A. 2001;98(4):1705-10.

63. Winterfeld G, Röser M. Disposition of ribosomal DNAs in the chromosomes of perennial oats (Poaceae: Aveneae). Bot J Linn Soc. 2007;155(2):193-210.

64. Bardsley D, Cuadrado A, Jack P, Harrison G, Castilho A, HeslopHarrison JS. Chromosome markers in the tetraploid wheat Aegilops ventricosa analysed by in situ hybridization. Theor Appl Genet. 1999;99(1-2):300-4.

65. Matzke MA, Mosher RA. RNA-directed DNA methylation: an epigenetic pathway of increasing complexity. Nat Rev Genet. 2014;15(6):394-408.

66. SanMiguel P, Vitte C. The LTR-retrotransposons of maize. In: Bennetzen J, Hake S, editors. Handbook of maize genetics and genomics. New York: Springer Verlag; 2009. p. 307-27.

67. Šmarda $P$, Bureš $P$, Horová $L$, Leitch I, Mucina L, Pacini E, et al. Ecological and evolutionary significance of genomic GC content diversity in monocots. Proc Natl Acad Sci U S A. 2014;111(39):E4096-102.

68. Shapiro JA. Mobile DNA and evolution in the $21^{\text {st }}$ century. Mob DNA. 2010;1(1):4.

69. Mravinac B, Plohl M. Parallelism in evolution of highly repetitive DNAs in sibling species. Mol Biol Evol. 2010;27(8):1857-67.

70. Zhong JS, Robbett M, Poire A, Preston JC. Successive evolutionary steps drove Pooideae grasses from tropical to temperate regions. New Phytol. 2018;217(2):925-38.

71. Lisch DR. Transposons in plant gene regulation. In: Fedoroff NV, editor. Plant transposons and genome dynamics in evolution. New Jersey: A John Wiley \& Sons, Inc.; 2013. p. 93-116.

72. Rozen S Skaletsky H. Primer3 on the WWW for general users and for biologist programmers. In: Misener S, Krawetz SA, editors. Bioinformatics methods and protocols. Totowa: Humana Press Inc; 1999. p. 365-86.

73. Schwarzacher T, Heslop-Harrison P. Practical in situ Hybridization. Oxford, UK BIOS Scientific Publishers; 2000

Ready to submit your research? Choose BMC and benefit from:

- fast, convenient online submission

- thorough peer review by experienced researchers in your field

- rapid publication on acceptance

- support for research data, including large and complex data types

- gold Open Access which fosters wider collaboration and increased citations

- maximum visibility for your research: over $100 \mathrm{M}$ website views per year

At $\mathrm{BMC}$, research is always in progress.

Learn more biomedcentral.com/submission 